\title{
REGULARITY OF SOLUTIONS IN PLASTICITY. II: PLATES
}

Abstract. The aim of this paper is to study the problem of regularity of displacement solutions in Hencky plasticity. We consider a plate made of a non-homogeneous material whose elastic-plastic properties change discontinuously. We prove that the displacement solutions belong to the space $W^{2,1}(\Omega)$ if the stress solution is continuous and belongs to the interior of the set of admissible stresses, at each point. The part of the functional which describes the work of boundary forces is relaxed.

1. Introduction. The principal aim of this contribution is to prove a theorem on regularity of displacement solutions in Hencky plasticity (see Theorem 24). We consider a plate made of a non-homogeneous Hencky material whose elastic-plastic properties change discontinuously.

In [6] and [15] the existence of solutions, for elastic-plastic plates, is proved in the space $S B H(\Omega)$ of Special Bounded Hessian. But the authors of those papers assume that the plate considered has elastic potential, i.e. the potential has nonlinear growth at infinity (cf. [6, (1) and (3)], [15, (2) and (6)]).

Demengel [7] solved the existence problem for an elastic-perfectly plastic plate made of a homogeneous and isotropic Hencky material. To prove that the functional of the total potential energy is weak* lower semicontinuous (l.s.c.) in the space $H B(\Omega)$, she used the method of relaxation of the kinematic boundary condition (see also [8], [14] and [3]).

In this contribution we show that the displacement solutions belong to the space $W^{2,1}(\Omega)$ if the stress solution is continuous and belongs to the $74 \mathrm{C} 05$.

2000 Mathematics Subject Classification: Primary 49N60; Secondary 49J45, 49K30, Hessian.

Key words and phrases: Hencky plasticity, displacement solution, space of bounded

Supported by the Committee for Scientific Research (Poland) grant No. 5P03A04620. 
interior of the set of admissible stresses, at each point. The part of the functional which describes the work of boundary forces is relaxed. We consider a non-homogeneous plate whose elastic-plastic properties change discontinuously.

2. Some basic definitions and theorems. In this paper $\Omega$ is a bounded, open, connected domain of class $C^{2}$ in $\mathbb{R}^{2}$. We use the notation from [4]. We define the following Banach space of functions with bounded Hessian $H B(\Omega)$ (see [7], [14]):

$$
H B(\Omega) \equiv\left\{u \in W^{1,1}(\Omega) \mid \frac{\partial^{2} u}{\partial x_{i} \partial x_{j}} \in \mathbb{M}_{b}(\Omega) \text { for all } i, j \in\{1,2\}\right\},
$$

with the natural norm

$$
\begin{aligned}
\|u\|_{H B(\Omega)} & =\|u\|_{W^{1,1}}+\|\nabla \nabla u\|_{\mathbb{M}_{b}\left(\Omega, \mathbf{E}_{s}^{2}\right)} \\
& =\|u\|_{W^{1,1}}+\sum_{i, j=1}^{2}\left\|\frac{\partial^{2} u}{\partial x_{i} \partial x_{j}}\right\|_{\mathbb{M}_{b}(\Omega, \mathbb{R})} .
\end{aligned}
$$

If $w \in D^{\prime}(\Omega)$ and $\nabla \nabla w \in \mathbb{M}_{b}\left(\Omega, \mathbf{E}_{s}^{2}\right)$, then $w \in H B(\Omega)$ (see [14]). Moreover, we define $\Pi_{2} \equiv \operatorname{ker} \nabla \nabla(\cdot)=\{u \in H B(\Omega) \mid \nabla \nabla u=0\}$.

Proposition 1 (see [14] and [3]). Let $\mathbf{g} \in D^{\prime}\left(\Omega, \mathbf{E}_{s}^{2}\right)$. Then the following conditions are equivalent:

(i) there exists $u \in D^{\prime}(\Omega)$ such that $\nabla \nabla u=\mathbf{g}$,

(ii) for all $\boldsymbol{\varphi} \in C_{\mathrm{c}}^{\infty}\left(\Omega, \mathbf{E}_{s}^{2}\right)$ with $\operatorname{div} \operatorname{div} \varphi=0, \sum_{i, j=1}^{2}\left\langle g_{i j} ; \varphi_{i j}\right\rangle_{D^{\prime} \times D}=0$.

Proposition 2 (see [14]). Let $H B(\Omega)$ and $L^{1}(\operatorname{Fr} \Omega)$ be endowed with the norm topologies. There exists a continuous surjective linear operator

$$
\left(\gamma_{0}, \gamma_{1}\right): H B(\Omega) \rightarrow \gamma_{0}\left(W^{2,1}(\Omega)\right) \times L^{1}(\operatorname{Fr} \Omega)
$$

such that

$$
\gamma_{0}(u)=u_{\mid \operatorname{Fr} \Omega}, \quad \gamma_{1}(u)=\frac{\partial u}{\partial \boldsymbol{\nu} \mid \operatorname{Fr} \Omega}
$$

for all $u \in C^{2}(\bar{\Omega})$, where $\boldsymbol{\nu}$ is the exterior unit vector normal to $\operatorname{Fr} \Omega$ and $\gamma_{0}\left(W^{2,1}(\Omega)\right)$ is endowed with the norm topology of $L^{1}(\operatorname{Fr} \Omega)$, since $\gamma_{0}\left(W^{2,1}(\Omega)\right) \subset L^{1}(\operatorname{Fr} \Omega)$.

We define spaces

$$
Z \equiv C_{\mathrm{c}}(\Omega, \mathbb{R}) \times C_{\mathrm{c}}\left(\Omega, \mathbf{E}_{s}^{2}\right), \quad Z_{0} \equiv\{(a, \mathbf{b}) \in Z \mid \operatorname{div} \operatorname{div} \mathbf{b}=-a\}
$$

endowed with the natural norm

$$
\begin{aligned}
\|(a, \mathbf{b})\|_{Z} & \equiv\|a\|_{C}+\|\mathbf{b}\|_{C} \\
& =\sup \{|a(x)| \mid x \in \Omega\}+\sup _{x \in \Omega} \sup _{i, j}\left\{\left|b_{i j}(x)\right| \mid x \in \Omega\right\}
\end{aligned}
$$


for $a \in C_{\mathrm{c}}(\Omega, \mathbb{R})$ and $\mathbf{b} \in C_{\mathrm{c}}\left(\Omega, \mathbf{E}_{s}^{2}\right)$. Then $H B(\Omega)$ is isomorphic to the dual of $\left[Z / Z_{0},\|\cdot\|_{Z}\right]$ (see $[3]$ and $[14]$ ). The topology $\sigma\left(\left(Z / Z_{0}\right)^{*} ; Z\right) \equiv$ $\sigma\left(H B(\Omega) ; C_{\mathrm{c}}(\Omega) \times C_{\mathrm{c}}\left(\Omega, \mathbf{E}_{s}^{2}\right)\right)$ is called the weak ${ }^{*} H B$ topology. A net $\left\{u_{\delta}\right\}_{\delta \in D} \subset H B(\Omega)$ is convergent to $u_{0} \in H B(\Omega)$ in this topology if and only if, for all $(a, \mathbf{b}) \in Z$,

$$
\int_{\Omega} a\left(u_{0}-u_{\delta}\right) d x+\int_{\Omega} \mathbf{b}: \nabla \nabla\left(u_{0}-u_{\delta}\right) \rightarrow 0 .
$$

For every $(v, w) \in \gamma_{0}\left(W^{2,1}(\Omega)\right) \times L^{1}(\operatorname{Fr} \Omega)$, the set $\{u \in H B(\Omega)$ $\gamma_{0}(u)=v$ and $\left.\gamma_{1}(u)=w\right\}$ is dense in the space $H B(\Omega)$ endowed with the weak* topology (see [2, Proposition 2.5] and [3]). Then the trace operator $\gamma_{0}: H B(\Omega) \rightarrow \gamma_{0}\left(W^{2,1}(\Omega)\right)$ (resp. $\gamma_{1}: H B(\Omega) \rightarrow L^{1}(\mathrm{Fr} \Omega)$ ) is not continuous on $\left[H B(\Omega)\right.$, weak ${ }^{*}$ topology] if the space $\gamma_{0}\left(W^{2,1}(\Omega)\right.$ ) (respectively $L^{1}(\operatorname{Fr} \Omega)$ ) is endowed with a Hausdorff topology (or a $T_{1}$-topology, see $[11])$.

Definition 1 (see [14] and [11, Chap. 1, Sec. 6]). A net $\left\{u_{\delta}\right\}_{\delta \in D}$ converges to $u_{0}$ in the topology $(2.8)-(2.9)$ if

$$
\begin{gathered}
u_{\delta} \rightarrow u_{0} \quad \text { in }\|\cdot\|_{W^{1, p}(\Omega)}, \text { where } 1 \leq p<2, \\
\nabla \nabla u_{\delta} \rightarrow \nabla \nabla u_{0} \quad \text { weak }^{*} \text { in } \mathbb{M}_{b}\left(\Omega, \mathbf{E}_{s}^{2}\right) .
\end{gathered}
$$

Proposition 3 (cf. [3]). The weak* HB topology and the (2.8)-(2.9) topology are equivalent on bounded subsets of $H B(\Omega)$.

Proof. Indeed, every bounded net $\left\{u_{\delta}\right\}_{\delta \in D}$ in $H B$ contains a finer net, convergent in the topology (2.8)-(2.9) (see [14]). Thus $\mathrm{cl}_{\|\cdot\|_{H B}} B(0, r)$ is a compact set in (2.8)-(2.9) and in the weak* $H B$ topology. Note that the weak $^{*} H B$ topology is weaker than the (2.8)-(2.9) topology. Moreover, among all Hausdorff topologies, compact topologies are minimal (see [11, Corollary 3.1.14]).

The weak* $H B(\Omega)$ topology and the topology (2.8)-(2.9) are not equivalent, because the trace $\gamma_{0}$, which is continuous in the topology (2.8)-(2.9), is not continuous in the weak ${ }^{*} H B$ topology. Let $\gamma_{0}\left(W^{2,1}(\Omega)\right)$ be endowed with the norm topology $L^{1}(\operatorname{Fr} \Omega)$. Then (for all $\left.r>0\right) \gamma_{0 \mid \mathrm{cl}_{\|\cdot\|_{H} B}} B(0, r)$ is a continuous function in the weak* $H B$ topology.

Proposition 4 (cf. [3, Theorem 5.13]). The injection of the space $\left[H B(\Omega)\right.$, weak $\left.{ }^{*}\right]$ into $\left[L^{p}(\Omega, \mathbb{R}),\|\cdot\|_{L^{p}}\right]$ is continuous on bounded subsets of $H B(\Omega)$, where $1 \leq p<2$.

We define the Banach space $W^{2}(\Omega$, div div) of measurable functions (cf. [14] and [3]):

$$
W^{2}(\Omega, \operatorname{div} \operatorname{div}) \equiv\left\{\mathbf{M} \in L^{\infty}\left(\Omega, \mathbf{E}_{s}^{2}\right) \mid \operatorname{div} \operatorname{div} \mathbf{M} \in L^{2}(\Omega, \mathbb{R})\right\}
$$


with the natural norm

$$
\|\mathbf{M}\|_{W^{2}(\Omega, \operatorname{div} \operatorname{div})}=\|\mathbf{M}\|_{L^{\infty}\left(\Omega, \mathbf{E}_{s}^{2}\right)}+\|\operatorname{div} \operatorname{div} \mathbf{M}\|_{L^{2}(\Omega)} .
$$

Moreover, $W_{\text {div }}^{2}\left(\Omega\right.$, div div) denotes the following subspace of $W^{2}(\Omega, \operatorname{div} \operatorname{div})$ : (2.12) $W_{\text {div }}^{2}(\Omega, \operatorname{div} \operatorname{div}) \equiv\left\{\mathbf{M} \in W^{2}(\Omega, \operatorname{div} \operatorname{div}) \mid \operatorname{div} \operatorname{div} \mathbf{M}=0\right.$ in $\left.\Omega\right\}$.

The distribution $\mathbf{M}: \nabla \nabla u$, where $\mathbf{M} \in W^{2}(\Omega$, div div), $u \in H B(\Omega)$, defined by

$$
\begin{aligned}
&\left\langle\mathbf{M}: \nabla \nabla u ; \varphi_{1}\right\rangle_{D^{\prime} \times D}=-\int_{\Omega} u \mathbf{M}: \nabla \nabla \varphi_{1} d x \\
&-2 \int_{\Omega} \mathbf{M}:\left((\nabla u) \otimes\left(\nabla \varphi_{1}\right)\right) d x+\int_{\Omega} u \varphi_{1}(\operatorname{div} \operatorname{div} \mathbf{M}) d x
\end{aligned}
$$

for $\varphi_{1} \in C_{\mathrm{c}}^{\infty}(\Omega)$, is a bounded measure on $\Omega$, absolutely continuous with respect to $|\nabla \nabla u|$ (see [14]).

TheOREM 5 (cf. [14]). Let $\gamma_{0}\left(W^{2,1}(\Omega)\right)$ be endowed with the norm

$$
\begin{aligned}
\gamma_{0}\left(W^{2,1}(\Omega)\right) \ni v & \mapsto \\
\|v\|_{0} & =\inf \left\{\|w\|_{W^{2,1}} \mid w \in W^{2,1}(\Omega) \text {, where } \gamma_{0}(w)=v\right\} .
\end{aligned}
$$

Moreover, let $\left(\gamma_{0}\left(W^{2,1}(\Omega)\right)\right)^{*}$ be endowed with the norm

$$
\left(\gamma_{0}\left(W^{2,1}(\Omega)\right)\right)^{*} \ni v^{*} \mapsto\left\|v^{*}\right\|_{0 *}=\sup \left\{\left|v^{*}(v)\right| \mid\|v\|_{0} \leq 1\right\} .
$$

Then there exists a continuous, linear, surjective map $\left(\beta_{0}, \beta_{1}\right)$ from $\left[W^{2}(\Omega\right.$, $\left.\operatorname{div} \operatorname{div}),\|\cdot\|_{W^{2}(\Omega, \operatorname{div} \operatorname{div})}\right]$ onto $\left[\left(\gamma_{0}\left(W^{2,1}(\Omega)\right)\right)^{*},\|\cdot\|_{0 *}\right] \times\left[L^{\infty}(\operatorname{Fr} \Omega),\|\cdot\|_{L^{\infty}}\right]$ such that

$$
\beta_{0}(\mathbf{M})=(\operatorname{div} \mathbf{M}) \boldsymbol{\nu}+\frac{\partial}{\partial s}((\mathbf{M} \boldsymbol{\nu}) \boldsymbol{\tau}), \quad \beta_{1}(\mathbf{M})=(\mathbf{M} \boldsymbol{\nu}) \boldsymbol{\nu}
$$

for every $\mathbf{M} \in C^{2}\left(\bar{\Omega}, \mathbf{E}_{s}^{2}\right)$, where $\boldsymbol{\nu}$ is the exterior unit vector normal to $\operatorname{Fr} \Omega$, $\boldsymbol{\tau}$ is a tangent unit vector and $s$ the curvilinear abscissa on $\operatorname{Fr} \Omega$ measured positively in the direction of $\boldsymbol{\tau}$. Furthermore, for all $u \in H B(\Omega)$ and $\mathbf{M} \in$ $W^{2}(\Omega$, div div), the following Green formula holds:

$$
\begin{aligned}
\int_{\Omega} \mathbf{M} & : \nabla \nabla u-\int_{\Omega} u(\operatorname{div} \operatorname{div} \mathbf{M}) d x \\
& =\int_{\operatorname{Fr} \Omega} \beta_{1}(\mathbf{M}) \gamma_{1}(u) d s-\left\langle\beta_{0}(\mathbf{M}) ; \gamma_{0}(u)\right\rangle_{\left(\gamma_{0}\left(W^{2,1}(\Omega)\right)\right)^{*} \times \gamma_{0}\left(W^{2,1}(\Omega)\right)} .
\end{aligned}
$$

Lemma 6 (cf. [3] and [14]). For every $\mathbf{M} \in W^{2}(\Omega$, div div), there exists a sequence $\left\{\mathbf{M}_{n}\right\}_{n \in \mathbb{N}} \subset W^{2}\left(\Omega\right.$, div div) $\cap C^{\infty}\left(\Omega, \mathbf{E}_{s}^{2}\right)$ such that (for all $n \in \mathbb{N}) \beta_{1}\left(\mathbf{M}_{n}\right)=\beta_{1}(\mathbf{M}), \beta_{0}\left(\mathbf{M}_{n}\right)=\beta_{0}(\mathbf{M})$ and

(2.18) $\quad \mathbf{M}_{n} \rightarrow \mathbf{M} \quad$ in the norm of $L^{p}\left(\Omega, \mathbf{E}_{s}^{2}\right)$, for all $p, 1 \leq p<\infty$, and weak ${ }^{*} L^{\infty}\left(\Omega, \mathbf{E}_{s}^{2}\right)$,

$$
\operatorname{div} \operatorname{div} \mathbf{M}_{n} \rightarrow \operatorname{div} \operatorname{div} \mathbf{M} \quad \text { in the norm of } L^{2}(\Omega) \text {. }
$$


Lemma 7 (see [3]). Let $\Omega_{1}$ be an open bounded set of class $C^{2}$. Let $\Omega$ be a bounded open connected set of class $C^{2}$ and suppose that $\Omega \subset \subset \Omega_{1} \subset \mathbb{R}^{2}$. For every $\mathbf{M} \in W^{2}\left(\Omega\right.$, div div), there exists $\mathbf{M}_{1} \in W^{2}\left(\Omega_{1}\right.$, div div) such that $\mathbf{M}_{1 \mid \Omega}=\mathbf{M}$ and $\beta_{0}\left(\mathbf{M}_{1}\right)=0, \beta_{1}\left(\mathbf{M}_{1}\right)=0$ on $\operatorname{Fr} \Omega_{1}$.

Proof. Let $\mathbf{M} \in W^{2}\left(\Omega\right.$, div div). By Theorem 5 , there exists $\mathbf{M}_{2} \in$ $W^{2}\left(\Omega_{1}-\bar{\Omega}, \operatorname{div} \operatorname{div}\right)$ such that $\beta_{0}(\mathbf{M})=-\beta_{0}\left(\mathbf{M}_{2}\right)$ and $\beta_{1}(\mathbf{M})=\beta_{1}\left(\mathbf{M}_{2}\right)$ on $\operatorname{Fr} \Omega$. Indeed, by [1] we can extend any function $t \in W^{2,1}(\Omega)$ to a function $\widehat{t} \in W^{2,1}\left(\Omega_{1}\right)$. Moreover, by Theorem 5 , the trace $\left(\beta_{0}, \beta_{1}\right)$ is a linear operator onto $\left[\gamma_{0}\left(W^{2,1}(\Omega)\right)\right]^{*} \times L^{\infty}(\operatorname{Fr} \Omega)$.

By Lemma 6 there exists $\mathbf{M}_{3} \in W^{2}\left(\Omega_{1}-\bar{\Omega}\right.$, div $\left.\operatorname{div}\right) \cap C^{\infty}\left(\Omega_{1}-\bar{\Omega}, \mathbf{E}_{s}^{2}\right)$ such that $\beta_{0}\left(\mathbf{M}_{2}\right)=\beta_{0}\left(\mathbf{M}_{3}\right)$ and $\beta_{1}\left(\mathbf{M}_{2}\right)=\beta_{1}\left(\mathbf{M}_{3}\right)$ on $\operatorname{Fr}\left(\Omega_{1}-\bar{\Omega}\right)$. Due to the Green formula (2.17), we find $\mathbf{M}_{4} \in W^{2}\left(\Omega_{1}\right.$, div div) such that

$$
\mathbf{M}_{4} \equiv \begin{cases}\mathbf{M} & \text { on } \Omega, \\ \mathbf{M}_{3} & \text { on } \Omega_{1}-\bar{\Omega}, \\ \beta_{1}(\mathbf{M}) \boldsymbol{\nu} \otimes \boldsymbol{\nu} & \text { on } \operatorname{Fr} \Omega,\end{cases}
$$

where $(\mathbf{p} \otimes \boldsymbol{\nu})_{i j}=p_{i} \nu_{j}$. If $h \in C_{\mathrm{c}}^{\infty}\left(\Omega_{1}, \mathbb{R}\right)$ and $h(x)=1$ for all $x \in \Omega$ then $\mathbf{M}_{1} \equiv h \mathbf{M}_{4}$.

3. Auxiliary theorems and spaces. In this paper the Lebesgue and Hausdorff measures on $\Omega$ and $\operatorname{Fr} \Omega$ are denoted by $d x$ and $d s$, respectively. Let $\Gamma_{0}$ and $\Gamma_{1}$ be Borel subsets of Fr $\Omega$ such that $\Gamma_{0} \cap \Gamma_{1}=\emptyset, \Gamma_{0} \cup \Gamma_{1}=$ Fr $\Omega$ and $\Gamma_{1}=\operatorname{cl}\left(\operatorname{int} \Gamma_{1}\right)$, where int $\Gamma_{1}$ denotes the interior of $\Gamma_{1}$ relative to the boundary. Let $\mathcal{K}_{p}: \bar{\Omega} \rightarrow 2^{\mathbf{E}_{s}^{2}}$ be a multifunction (defined for every $x \in \bar{\Omega}$ ) and suppose there exist $r_{2}>r_{1}>0$ such that for every $x \in \bar{\Omega}$,

$$
B_{\mathbf{E}_{s}^{2}}\left(0, r_{1}\right) \subset \mathcal{K}_{p}(x) \subset B_{\mathbf{E}_{s}^{2}}\left(0, r_{2}\right) .
$$

The set $\mathcal{K}_{p}(x)$ is the elasticity convex domain at the point $x$.

Assumption 1 (cf. [4]). Let $\Omega$ and $\Omega_{1}\left(\Omega \subset \subset \Omega_{1}\right)$ be bounded open connected sets of class $C^{2}$ in $\mathbb{R}^{2}$ and suppose the inclusion (3.1) holds. For every $y \in \bar{\Omega}$,

$$
\begin{aligned}
\mathcal{K}_{p}(y)=\left\{\mathbf{z}(y) \mid \mathbf{z} \in C^{1}\left(\bar{\Omega}, \mathbf{E}_{s}^{2}\right), \mathbf{z}_{\mid \operatorname{int} \Omega} \in W^{2}(\Omega, \operatorname{div} \operatorname{div}),\right. \\
\left.\mathbf{z}(x) \in \mathcal{K}_{p}(x) \text { for } d x \text {-a.e. } x \in \Omega\right\} .
\end{aligned}
$$

Moreover, for all $x \in \bar{\Omega}, \mathcal{K}_{p}(x)$ is a convex and closed subset in $\mathbf{E}_{s}^{2}$.

Assumption 2. There exist $\bar{a}>0,\left[q_{i j}\right] \in L^{\infty}\left(\Omega, \mathbf{E}_{s}^{2}\right)$ and $a_{i j k l} \in$ $L^{\infty}(\Omega, \mathbb{R})$ for $i, j, k, l \in\{1,2\}$ such that

$$
\sum_{i, j, k, l=1}^{2} a_{i j k l}(x) w_{i j}^{*} w_{k l}^{*}>\bar{a}\left\|\left[w_{i j}^{*}\right]\right\|_{\mathbf{E}_{s}^{2}}^{2},
$$




$$
j^{*}\left(x,\left[w_{i j}^{*}\right]\right)=\sum_{i, j, k, l=1}^{2} a_{i j k l}(x)\left(w_{i j}^{*}-q_{i j}(x)\right)\left(w_{k l}^{*}-q_{k l}(x)\right)+I_{\mathcal{K}(x)}\left(\left[w_{i j}^{*}\right]\right)
$$

for $d x$-a.e. $x \in \Omega$ and for every $\left[w_{i j}^{*}\right] \in \mathbf{E}_{s}^{2}$, where $w_{i j}^{*}$ and $w_{k l}^{*}$ are components of the matrix $\left[w_{i j}^{*}\right]$.

Assumption 3. Let $\Gamma_{1}=\operatorname{Fr} \Omega \cap \mathcal{C}$, where $\mathcal{C}=\operatorname{cl} \operatorname{int} \mathcal{C} \subset \Omega_{1}$ is a closed Caccioppoli set and $d s(\operatorname{Fr} \Omega \cap \operatorname{Fr} \mathcal{C})=0$ (cf. [12] and [4]).

We define

$$
j(x, \mathbf{w}) \equiv j^{* *}(x, \mathbf{w}) \equiv \sup \left\{\mathbf{w}: \mathbf{w}^{*}-j^{*}\left(x, \mathbf{w}^{*}\right) \mid \mathbf{w}^{*} \in \mathbf{E}_{s}^{2}\right\}
$$

for $d x$-a.e. $x \in \Omega$ and all $\mathbf{w} \in \mathbf{E}_{s}^{2}$. The function $j$ is a convex normal integrand (cf. [10, Chap. VIII, Proposition 1.2]). By (3.1) and (3.4) there exists $k>0$ such that

$$
c r_{1}\|\mathbf{w}\|_{\mathbf{E}_{s}^{2}}-k \leq j(x, \mathbf{w}) \quad \text { for } d x \text {-a.e. } x \in \Omega
$$

(cf. [4, (3.5)]). Define $j_{\infty}: \bar{\Omega} \times \mathbf{E}_{s}^{2} \rightarrow \mathbb{R} \cup\{+\infty\}$ by

$$
j_{\infty}(x, \mathbf{w}) \equiv \sup \left\{\mathbf{w}: \mathbf{w}^{*}-I_{\mathcal{K}_{p}(x)}\left(\mathbf{w}^{*}\right) \mid \mathbf{w}^{*} \in \mathbf{E}_{s}^{2}\right\}
$$

for $x \in \bar{\Omega}$ and $\mathbf{w} \in \mathbf{E}_{s}^{2}$. Because of (3.1) we have, for all $x \in \bar{\Omega}$ (see [4, $(3.7)])$

$$
c_{1} r_{1}\|\mathbf{w}\|_{\mathbf{E}_{s}^{2}} \leq j_{\infty}(x, \mathbf{w}) \leq c_{2} r_{2}\|\mathbf{w}\|_{\mathbf{E}_{s}^{2}} .
$$

4. Regularity of displacement solutions. In this section we prove that the displacement solutions of the relaxed functional belong to the space $W^{2,1}(\Omega)$ if the stress solution is continuous and belongs to the interior of the set of admissible stresses, at each point (see Theorem 24 ).

Let $\mathcal{Z}: H B(\Omega) \rightarrow \mathbb{M}_{b}\left(\Omega, \mathbf{E}_{s}^{2}\right) \times L^{1}(\operatorname{Fr} \Omega, \mathbb{R}) \times C(\operatorname{Fr} \Omega)$ be given by

$$
H B(\Omega) \ni u \mapsto \mathcal{Z}(u)=\left((\nabla \nabla u), \gamma_{1}(u), \gamma_{0}(u)\right) .
$$

We define spaces

$$
\mathcal{Z}(H B(\Omega)) \equiv\left\{\left(\nabla \nabla u, \gamma_{1}(u), \gamma_{0}(u)\right) \mid u \in H B(\Omega)\right\}
$$

and

$$
W^{2}(\Omega, \operatorname{div} \operatorname{div}) \times C(\operatorname{Fr} \Omega),
$$

put in duality by the bilinear pairing $\langle\cdot ; \cdot\rangle_{Z}$, where

$$
\begin{aligned}
&\left\langle\left(\nabla \nabla u, \gamma_{1}(u), \gamma_{0}(u)\right) ;\left(\varphi^{*}, \psi^{*}\right)\right\rangle_{Z} \\
&=\int_{\Omega} \varphi^{*}: \nabla \nabla u-\int_{\operatorname{Fr} \Omega} \beta_{1}\left(\varphi^{*}\right) \gamma_{1}(u) d s+\int_{\operatorname{Fr} \Omega} \gamma_{0}(u) \psi^{*} d s
\end{aligned}
$$

for every $\varphi^{*} \in W^{2}(\Omega, \operatorname{div} \operatorname{div})$ and

$$
\psi^{*} \in C(\operatorname{Fr} \Omega, \mathbb{R}), \quad\left(\nabla \nabla u, \gamma_{1}(u), \gamma_{0}(u)\right) \in \mathcal{Z}(H B(\Omega)) .
$$


Moreover, we define a subspace $C_{\operatorname{div} \operatorname{div}}^{1}\left(\bar{\Omega}, \mathbf{E}_{s}^{2}\right)$ of $W^{2}(\Omega, \operatorname{div} \operatorname{div})$ by

$$
C_{\operatorname{div} \operatorname{div}}^{1}\left(\bar{\Omega}, \mathbf{E}_{s}^{2}\right) \equiv\left\{\boldsymbol{\varphi}^{*} \in C^{1}\left(\bar{\Omega}, \mathbf{E}_{s}^{2}\right) \mid \boldsymbol{\varphi}_{\mid \Omega}^{*} \in W^{2}(\Omega, \operatorname{div} \operatorname{div})\right\} .
$$

REMARK 1. The definition of spaces in duality requires that for every $\left(\boldsymbol{\varphi}^{*}, \psi^{*}\right) \in W^{2}(\Omega, \operatorname{div} \operatorname{div}) \times C(\operatorname{Fr} \Omega),\left(\boldsymbol{\varphi}^{*}, \psi^{*}\right) \neq(\mathbf{0}, 0)$, there exists $\left(\nabla \nabla u, \gamma_{1}(u), \gamma_{0}(u)\right) \in \mathcal{Z}(H B(\Omega))$ such that

$$
\left\langle\left(\nabla \nabla u, \gamma_{1}(u), \gamma_{0}(u)\right) ;\left(\varphi^{*}, \psi^{*}\right)\right\rangle_{Z} \neq 0 .
$$

But for every $\varphi_{s}^{*} \in W^{2}(\Omega$, div div $)$ such that $\operatorname{div} \operatorname{div} \varphi_{s}^{*}=0$ in $\Omega, \beta_{0}\left(\varphi_{s}^{*}\right)=0$ on $\operatorname{Fr} \Omega$, and for every $\left(\nabla \nabla u, \gamma_{1}(u), \gamma_{0}(u)\right) \in \mathcal{Z}(H B(\Omega))$,

$$
\left\langle\left((\nabla \nabla u), \gamma_{1}(u), \gamma_{0}(u)\right) ;\left(\varphi_{s}^{*}, 0\right)\right\rangle_{Z}=\int_{\Omega} u\left(\operatorname{div} \operatorname{div} \varphi_{s}^{*}\right) d x=0
$$

(see (2.17) and [4]). We do not get a contradiction, since we do not use the Hausdorff property of the topology $\sigma\left(W^{2}(\Omega, \operatorname{div} \operatorname{div}) \times C(\operatorname{Fr} \Omega) ; \mathcal{Z}(H B(\Omega))\right)$ (cf. [4, Remark 3]).

Let the bilinear form $\langle\cdot ; \cdot\rangle_{b}$ between $\mathcal{Z}(H B(\Omega))$ and $W^{2}(\Omega$, div div) be given by

$$
\begin{aligned}
& \left\langle\left(\nabla \nabla u, \gamma_{1}(u), \gamma_{0}(u)\right) ;\left(\varphi^{*}, \beta_{0}\left(\varphi^{*}\right)\right)\right\rangle_{b}=\int_{\Omega} \varphi^{*}:(\nabla \nabla u) \\
& \quad-\int_{\operatorname{Fr} \Omega} \beta_{1}\left(\varphi^{*}\right) \gamma_{1}(u) d s+\left\langle\beta_{0}\left(\varphi^{*}\right) ; \gamma_{0}(u)\right\rangle_{\left(\gamma_{0}\left(W^{2,1}\right)\right)^{*} \times \gamma_{0}\left(W^{2,1}\right)}
\end{aligned}
$$

for $u \in H B(\Omega)$ and $\varphi^{*} \in W^{2}\left(\Omega\right.$, div div). A net $\left\{u_{\delta}\right\}_{\delta \in D} \subset H B(\Omega)$ is said to converge to $\widehat{u} \in H B(\Omega)$ in the topology $\sigma\left(\mathcal{Z}(H B(\Omega)) ; W^{2}(\Omega\right.$, div div $\left.)\right)$ if

$$
\left\langle\left(\nabla \nabla\left(u_{\delta}-\widehat{u}\right), \gamma_{1}\left(u_{\delta}-\widehat{u}\right), \gamma_{0}\left(u_{\delta}-\widehat{u}\right)\right) ;\left(\varphi^{*}, \beta_{0}\left(\varphi^{*}\right)\right)\right\rangle_{b} \rightarrow 0
$$

for all $\varphi^{*} \in W^{2}(\Omega, \operatorname{div} \operatorname{div})$.

TheOREM 8. Every closed ball $\mathrm{cl}_{\|\cdot\|_{H B}}(B(0, \widehat{r}))($ in $\mathcal{Z}(H B(\Omega)))$ is compact in the topology $\sigma\left(\mathcal{Z}(H B(\Omega)) ; W^{2}(\Omega\right.$, div div $\left.)\right)$.

Proof. Step 1. Let a net $\left\{u_{\delta}\right\}_{\delta \in D} \subset H B(\Omega)$ be included in $B_{H B}(0, \widehat{r})$. The injection $H B(\Omega) \subset H^{1}(\Omega)$ is continuous and injection $H^{1}(\Omega)$ into $L^{2}(\Omega)$ is compact (see [14, Chap. 3, (2.77)], [14, Chap. 1, (1.21)] and [1, Theorem 6.2]). Then there exists a finer net $\left\{u_{\delta_{\alpha}}\right\}_{\alpha \in A} \subset\left\{u_{\delta}\right\}_{\delta \in D}$ and a function $u_{1} \in L^{2}(\Omega)$ such that

$$
\left\|u_{\delta_{\alpha}}-u_{1}\right\|_{L^{2}(\Omega, \mathbb{R})} \rightarrow 0
$$

and

$$
\begin{aligned}
&\left\langle\left(\nabla \nabla u_{\delta_{\alpha}}, \gamma_{1}\left(u_{\delta_{\alpha}}\right), \gamma_{0}\left(u_{\delta_{\alpha}}\right)\right) ;\left(\varphi^{*}, \beta_{0}\left(\varphi^{*}\right)\right)\right\rangle_{b} \\
&=\int_{\Omega} u_{\delta_{\alpha}}\left(\operatorname{div} \operatorname{div} \varphi^{*}\right) d x \rightarrow \int_{\Omega} u_{1}\left(\operatorname{div} \operatorname{div} \varphi^{*}\right) d x
\end{aligned}
$$


for every $\varphi^{*} \in W^{2}(\Omega, \operatorname{div} \operatorname{div})$, because $\operatorname{div} \operatorname{div} \varphi^{*} \in L^{2}(\Omega)$ (cf. (2.17)). Since $\Omega$ is bounded subset of $\mathbb{R}^{2}$ and (4.11) holds, we have $\left\|u_{\delta_{\alpha}}-u_{1}\right\|_{L^{1}(\Omega, \mathbf{R})} \rightarrow 0$.

Step 2. By the compactness theorem for the space $B D(\Omega)$ (see $[14$, Chap. 2, Theorem 2.4]) the injection $\left\{\nabla u \in L^{1}\left(\Omega, \mathbb{R}^{2}\right) \mid u \in H B(\Omega)\right\}$ into $L^{1}\left(\Omega, \mathbb{R}^{2}\right)$ is compact. Then there exists a finer net $\left\{u_{\delta_{\alpha_{\beta}}}\right\}_{\beta \in B}$ and a function $\widehat{w} \in L^{1}\left(\Omega, \mathbb{R}^{2}\right)$ such that $\left\|\nabla u_{\delta_{\alpha_{\beta}}}-\widehat{w}\right\|_{L^{1}\left(\Omega, \mathbb{R}^{2}\right)} \rightarrow 0$. We obtain $-\int_{\Omega} u_{\delta_{\alpha_{\beta}}} \operatorname{div} \xi d x=\int_{\Omega} \nabla u_{\delta_{\alpha_{\beta}}} \xi d x \rightarrow \int_{\Omega} \widehat{w} \xi d x$ for every $\xi \in C_{\mathrm{c}}^{1}\left(\Omega, \mathbb{R}^{2}\right)$. Then $\nabla u_{1}=\widehat{w}$ in the sense of distributions on $\Omega$. Therefore, by Step 1, we obtain

$$
\left\|u_{\delta_{\alpha_{\beta}}}-u_{1}\right\|_{W^{1,1}(\Omega)} \rightarrow 0 .
$$

Step 3. The net $\left\{u_{\delta_{\alpha_{\beta}}}\right\}_{\beta \in B}$ is bounded in $H B(\Omega)$, so there exists a finer net $\left\{u_{\delta_{\alpha_{\beta} \gamma}}\right\}$ and a measure $\boldsymbol{\mu}_{1} \in \mathbb{M}_{b}\left(\Omega, \mathbf{E}_{s}^{2}\right)$ such that $\int_{\Omega} \varphi:\left(\nabla \nabla u_{\delta_{\alpha_{\beta}}}\right) \rightarrow$ $\int_{\Omega} \boldsymbol{\varphi}: \boldsymbol{\mu}_{1}$ for every $\boldsymbol{\varphi} \in C_{\mathrm{c}}^{2}\left(\Omega, \mathbf{E}_{s}^{2}\right)$. Since we have $\int_{\Omega}(\operatorname{div} \operatorname{div} \boldsymbol{\varphi}) u_{\delta_{\alpha_{\beta_{\gamma}}}} d x \rightarrow$ $\int_{\Omega}(\operatorname{div} \operatorname{div} \varphi) u_{1} d x=\int_{\Omega} \boldsymbol{\varphi}: \boldsymbol{\mu}_{1}$ for such $\boldsymbol{\varphi}$, we find that $\nabla \nabla u_{1}=\boldsymbol{\mu}_{1}$ in the sense of distributions on $\Omega$. Therefore, by [14, Chap. 3, (2.79)], $u_{1} \in H B(\Omega)$ and $\left\{u_{\delta_{\alpha_{\beta}}}\right\}$ converges to $u_{1}$ in $\sigma\left(\mathcal{Z}(H B(\Omega)) ; W^{2}(\Omega\right.$, div div)) (cf. (4.9) and (4.10)).

Step 4. We show that $\left\|u_{1}\right\|_{H B} \leq \widehat{r}$. Indeed, $\lim \left\|u_{\delta_{\alpha_{\beta} \gamma}}\right\|_{W^{1,1}(\Omega)}=$ $\left\|u_{1}\right\|_{W^{1,1}(\Omega)}$ by (4.13). Then for every $\varepsilon>0$ there exists $\gamma_{\varepsilon}$ such that for every $\gamma>\gamma_{\varepsilon}$ we obtain

$$
\begin{aligned}
& \quad \widehat{r}-\lim \left\|u_{\delta_{\alpha_{\beta_{\gamma}}}}\right\|_{W^{1,1}}+\varepsilon \geq \sup _{\gamma>\gamma_{\varepsilon}}\left\|\nabla \nabla u_{\delta_{\alpha_{\beta_{\gamma}}}}\right\|_{\mathbb{M}_{b}\left(\Omega, \mathbf{E}_{s}^{2}\right)} \\
& =\sup _{\gamma>\gamma_{\varepsilon}} \sup _{\mathbf{w} \in C_{0}}\left\{\int_{\Omega} \mathbf{w}: \nabla \nabla u_{\delta_{\alpha_{\beta_{\gamma}}}} \mid \mathbf{w} \in C_{0}\left(\Omega, \mathbf{E}_{s}^{2}\right), \sup _{i, j}\left\{\left\|w_{i j}\right\|_{C(\Omega)}\right\} \leq 1\right\} \\
& =\sup _{\mathbf{w} \in C_{0}} \sup _{\gamma>\gamma_{\varepsilon}}\left\{\int_{\Omega} \mathbf{w}: \nabla \nabla u_{\delta_{\alpha_{\beta}}} \mid \mathbf{w} \in C_{0}\left(\Omega, \mathbf{E}_{s}^{2}\right), \sup _{i, j}\left\{\left\|w_{i j}\right\|_{C(\Omega)}\right\} \leq 1\right\} \\
& \geq \sup _{\mathbf{w} \in C_{0}} \lim _{\gamma>\gamma_{\varepsilon}}\left\{\int_{\Omega} \mathbf{w}: \nabla \nabla u_{\delta_{\alpha_{\beta_{\gamma}}}} \mid \mathbf{w} \in C_{0}\left(\Omega, \mathbf{E}_{s}^{2}\right), \sup _{i, j}\left\{\left\|w_{i j}\right\|_{C(\Omega)}\right\} \leq 1\right\} \\
& =\sup _{\mathbf{w} \in C_{0}}\left\{\int_{\Omega} \mathbf{w}: \nabla \nabla u_{1} \mid \mathbf{w} \in C_{0}\left(\Omega, \mathbf{E}_{s}^{2}\right), \sup _{i, j}\left\{\left\|w_{i j}\right\|_{C(\Omega)}\right\} \leq 1\right\} \\
& =\left\|\nabla \nabla u_{1}\right\|_{\mathbb{M}_{b}\left(\Omega, \mathbf{E}_{s}^{2}\right)},
\end{aligned}
$$

where $w_{i j}$ are the components of the matrix $\mathbf{w}$ (cf. definition of $\|\cdot\|_{\mathbb{M}_{b}\left(\Omega, \mathbf{E}_{s}^{2}\right)}$ ). Therefore $\left[\operatorname{cl}_{\|\cdot\|_{H B}}(B(0, \widehat{r})), \sigma\left(\mathcal{Z}(H B(\Omega)) ; W^{2}(\Omega, \operatorname{div} \operatorname{div})\right)\right]$ is compact. 
Theorem 9 (cf. [3]). The space $\left[\mathrm{cl}_{\|\cdot\|_{H B}} B(0, \widehat{r})\right.$, weak ${ }^{*} H B$ topology] is homeomorphic to the space $\mathcal{Z}\left(\mathrm{cl}_{\|\cdot\|_{H B}} B(0, r)\right)$ endowed with the topology

$$
\sigma\left(\mathcal{Z}(H B(\Omega)) ; C_{\operatorname{div} \operatorname{div}}^{1}\left(\bar{\Omega}, \mathbf{E}_{s}^{2}\right) \times C(\operatorname{Fr} \Omega, \mathbb{R})\right),
$$

where the bilinear form $\langle\cdot ; \cdot\rangle_{Z}$ between $\mathcal{Z}(H B(\Omega))$ and $C_{\operatorname{div} \operatorname{div}}^{1}\left(\bar{\Omega}, \mathbf{E}_{s}^{2}\right) \times$ $C(\operatorname{Fr} \Omega, \mathbb{R})$ is given by (4.4) and (4.5) (see also (4.6)).

Proof. $\mathcal{Z}$ is a one-to-one function. We will show that $\mathcal{Z}\left(\mathrm{cl}_{\|\cdot\|_{H B}} B(0, \widehat{r})\right)$ is compact in the topology (4.15).

Step 1. Let a net $\left\{u_{t}\right\}_{t \in T}$ be a subset of $\operatorname{cl}_{\|\cdot\|_{H B}} B(0, \widehat{r})$ (cf. [11, pp. $73-$ $81])$. Due to Steps 1 and 2 of the proof of Theorem 8 , there exists a finer net $\left\{u_{\delta}\right\}_{\delta \in D}$ (where $D \subset T$ ) and $u_{1} \in W^{1,1}(\Omega)$ such that $\left\|u_{\delta}-u_{1}\right\|_{W^{1,1}(\Omega)} \rightarrow 0$ and $\left\|u_{\delta}-u_{1}\right\|_{L^{2}(\Omega, \mathbb{R})} \rightarrow 0$.

Step 2. The net $\left\{\nabla \nabla u_{\delta}\right\}_{\delta \in D}$ is bounded in $\|\cdot\|_{\mathbb{M}_{b}}$. Thus there exists a finer net $\left\{\nabla \nabla u_{\kappa}\right\}_{\kappa \in K}($ where $K \subset D)$ such that $\nabla \nabla u_{\kappa} \rightarrow \mathbf{M}_{0} \in \mathbb{M}_{b}\left(\Omega, \mathbf{E}_{s}^{2}\right)$ (in the weak* $\mathbb{M}_{b}$ topology). By Proposition 1, there exists $u_{g} \in D^{\prime}(\Omega)$ such that $\nabla \nabla u_{g}=\mathbf{M}_{0}$, and $\nabla \nabla u_{\kappa} \rightarrow \nabla \nabla u_{g} \in \mathbb{M}_{b}\left(\Omega, \mathbf{E}_{s}^{2}\right)$ in the weak* $\mathbb{M}_{b}\left(\Omega, \mathbf{E}_{s}^{2}\right)$ topology. The net $\left\{u_{\kappa}\right\}_{\kappa \in K}$ is finer than $\left\{u_{\delta}\right\}_{\delta \in D}$, so $u_{1}=u_{g}+c$, where $c \in \operatorname{ker}(\nabla \nabla(\cdot))$, because

$$
\begin{aligned}
& \int_{\Omega} \xi: \nabla \nabla u_{\kappa}=\int_{\Omega}(\operatorname{div} \operatorname{div} \xi) u_{\kappa} d x \rightarrow \int_{\Omega}(\operatorname{div} \operatorname{div} \xi) u_{g} d x \\
& \forall \xi \in C_{\mathrm{c}}^{2}\left(\Omega, \mathbf{E}_{s}^{2}\right) .
\end{aligned}
$$

Therefore, we can assume that $u_{1}=u_{g}$ and we conclude that $u_{g} \in H B(\Omega)$.

Step 3. By Step 1 of the proof of Theorem 8 we get

$$
\left\langle\left(\nabla \nabla\left(u_{\kappa}-u_{g}\right), \gamma_{1}\left(u_{\kappa}-u_{g}\right), \gamma_{0}\left(u_{\kappa}-u_{g}\right)\right) ;\left(\varphi^{*}, \beta_{0}\left(\varphi^{*}\right)\right)\right\rangle_{b} \rightarrow 0
$$

for every $\varphi^{*} \in C_{\operatorname{div} \operatorname{div}}^{1}\left(\bar{\Omega}, \mathbf{E}_{s}^{2}\right)$, since $C_{\operatorname{div} \operatorname{div}}^{1}\left(\bar{\Omega}, \mathbf{E}_{s}^{2}\right) \subset W^{2}(\Omega$, div div $)$. Moreover, $\left\|\gamma_{0}\left(u_{\kappa}-u_{g}\right)\right\|_{L^{1}(\mathrm{Fr} \Omega)} \rightarrow 0$, because $\left\|u_{\kappa}-u_{g}\right\|_{W^{1,1}(\Omega)} \rightarrow 0$ (cf. Step 1). Then

$$
\int_{\operatorname{Fr} \Omega} \gamma_{0}\left(u_{\kappa}-u_{g}\right) \psi^{*} d s \rightarrow 0
$$

for every $\psi^{*} \in C(\operatorname{Fr} \Omega, \mathbb{R})$, since the closed and bounded set $\operatorname{Fr} \Omega$ is compact and $C(\operatorname{Fr} \Omega, \mathbb{R}) \subset L^{\infty}(\operatorname{Fr} \Omega, \mathbb{R})$.

Step 4. Because of Step 1, we can show (similarly to Step 4 of the proof of Theorem 8) that $\left\|u_{g}\right\|_{H B} \leq \widehat{r}$.

Step 5. By $(2.16), \beta_{0}\left(\varphi^{*}\right) \in C(\operatorname{Fr} \Omega, \mathbb{R})$ if $\varphi^{*} \in C_{\operatorname{div} \operatorname{div}}^{1}\left(\bar{\Omega}, \mathbf{E}_{s}^{2}\right)$. Then

$$
\begin{aligned}
\int_{\Omega} \varphi^{*}: \nabla \nabla\left(u_{\kappa}-u_{g}\right)-\int_{\operatorname{Fr} \Omega} \beta_{1}\left(\varphi^{*}\right) \gamma_{1}\left(u_{\kappa}-u_{g}\right) d s & \\
& +\int_{\operatorname{Fr} \Omega} \gamma_{0}\left(u_{\kappa}-u_{g}\right) \psi^{*} d s \rightarrow 0
\end{aligned}
$$


for every $\varphi^{*} \in C_{\text {div div }}^{1}\left(\bar{\Omega}, \mathbf{E}_{s}^{2}\right)$ and $\psi^{*} \in C(\operatorname{Fr} \Omega, \mathbb{R})$ (see (4.4), (4.9), (4.17) and (4.18)). By Steps 1 and 2 we obtain

$$
\int_{\Omega} \mathbf{M}: \nabla \nabla\left(u_{\kappa}-u_{g}\right)+\int_{\Omega} h\left(u_{\kappa}-u_{g}\right) d x \rightarrow 0
$$

for every $\mathbf{M} \in C_{\mathrm{c}}\left(\Omega, \mathbf{E}_{s}^{2}\right)$ and $h \in C_{\mathrm{c}}(\Omega, \mathbb{R})$, because $\nabla \nabla u_{\kappa} \rightarrow \nabla \nabla u_{g}$ (in the weak* $\mathbb{M}_{b}$ topology) and $\left\|u_{\kappa}-u_{g}\right\|_{L^{2}(\mathrm{Fr} \Omega)} \rightarrow 0$. Therefore the set $\operatorname{cl}_{\|\cdot\|_{H B}} B(0, \widehat{r})$ (endowed with the topology where convergence of nets is defined by (4.19) and (4.20)) is compact. The Hausdorff topologies (4.15) and weak* $H B$ are weaker than the topology given by (4.19)-(4.20). By [11, Corollary 3.1.14] the weak* $H B$ topology and (4.15) are homeomorphic on the set $\mathrm{cl}_{\|\cdot\|_{H B}} B(0, \widehat{r})$.

THEOREM 10. Every closed ball $\mathrm{cl}_{\|\cdot\|_{H B}} B(0, \widehat{r})$ endowed with the topology $\sigma\left(\mathcal{Z}(H B(\Omega)) ; W^{2}(\Omega, \operatorname{div} \operatorname{div})\right)$ is homeomorphic to $\left[\operatorname{cl}_{\|\cdot\|} B_{H B}(0, \widehat{r})\right)$; weak* $H B$ topology].

Proof. Similarly to Step 5 of the proof of Theorem 9, we define a compact topology on $\mathrm{cl}_{\|\cdot\|_{H B}} B(0, \widehat{r})$, which is stronger than the weak* $H B$ topology and $\sigma\left(\mathcal{Z}(H B(\Omega)) ; W^{2}(\Omega\right.$, div div $\left.)\right)$. Next we use Corollary 3.1.14 of [11] to deduce the equivalence of the three topologies on $\mathrm{cl}_{\|\cdot\|_{H B}} B(0, \widehat{r})$.

We study a thin plate occupying a given surface $\Omega$. This plate is subjected to a force, of surface density $f \in L^{2}(\Omega, \mathbb{R})$, acting in a direction at right angles to the surface. We shall suppose the plate to be subject to force $g_{0} \in L^{\infty}(\operatorname{Fr} \Omega) \subset\left(\gamma_{0}\left(W^{2,1}(\Omega)\right)\right)^{*}$ on $\Gamma_{1}$ and bending moment of linear density $g_{1} \in L^{\infty}\left(\Gamma_{1}, \mathbb{R}\right)$. Let $\lambda$ (where $\infty>\lambda \geq 0$ ) stand for the load multiplier.

Define

$$
\begin{aligned}
& W^{2}(\Omega, \operatorname{div} \operatorname{div}) \ni \mathbf{M} \mapsto\left\lceil P_{\lambda, r}^{*}\right\rceil(\mathbf{M})=-\int_{\Gamma_{1}} I_{B_{\mathbb{R}}(0, r)}\left(\lambda g_{1}-\beta_{1}(\mathbf{M})\right) d s \\
& -I_{B_{\left(\gamma_{0}\left(W^{2,1}\right)\right)^{*}}(0, r)}\left(\beta_{0}(\mathbf{M})_{\mid \operatorname{int} \Gamma_{1}}-\lambda g_{0}\right)-\int_{\Omega} j^{*}(x, \mathbf{M}) d x
\end{aligned}
$$

if $\operatorname{div} \operatorname{div} \mathbf{M}=+\lambda f$ in $\Omega$ and $\left\lceil P_{\lambda, r}^{*}\right\rceil(\mathbf{M})=-\infty$ otherwise. Here $\beta_{0}(\mathbf{M})_{\mid \operatorname{int} \Gamma_{1}}$ denotes the functional $\widetilde{\psi}^{*} \in\left(\gamma_{0}\left(W^{2,1}(\Omega)\right)\right)^{*}$ with $\widetilde{\psi}^{*}=\beta_{0}(\mathbf{M})$ on int $\Gamma_{1}$ and $\widetilde{\psi}^{*}=0$ on $\operatorname{cl} \Gamma_{0}$ (see [3, Proposition 5.16]). Moreover, $I_{A}(\cdot)$ denotes the indicator function of a set $A$. The dual relaxed problem, for $r \geq 0$, is given by the formula

$$
\begin{aligned}
& \left(P_{\lambda, r}^{*}\right) \text { find } \sup \left\{\left\lceil P_{\lambda, r}^{*}\right\rceil(\mathbf{M}) \mid \mathbf{M} \in W^{2}(\Omega, \operatorname{div} \operatorname{div}),\right. \\
& \qquad \operatorname{div} \operatorname{div} \mathbf{M}=\lambda f \text { in } \Omega\} .
\end{aligned}
$$


Assumption 4. There exist $\mathbf{M}_{L} \in C_{\text {div div }}^{1}\left(\bar{\Omega}, \mathbf{E}_{s}^{2}\right)$ such that $\left\lceil P_{\lambda, r}^{*}\right\rceil\left(\mathbf{M}_{L}\right)$ $=\sup \left(P_{\lambda, r}^{*}\right)>-\infty, \beta_{1}\left(\mathbf{M}_{L}\right)=\lambda g_{1}$ on $\Gamma_{1}, \beta_{0}\left(\mathbf{M}_{L}\right)=\lambda g_{0}$ on $\Gamma_{1}$ and $\mathbf{M}_{L}(x) \in \mathcal{K}_{p}(x)$ for all $x \in \Omega$. Moreover, there exist $\delta_{0}>0$ such that $\operatorname{dist}\left(\mathbf{M}_{L}(x), \operatorname{Fr} \mathcal{K}_{p}(x)\right)=\inf \left\{\left\|\mathbf{M}_{L}(x)-\mathbf{z}\right\|_{\mathbf{E}_{s}^{2}} \mid \mathbf{z} \in \operatorname{Fr} \mathcal{K}_{p}(x)\right\}>\delta_{0}$ for every $x \in \Omega$.

Define $H_{r}: \mathcal{Z}(H B) \rightarrow \mathbb{R} \cup\{+\infty\}$ by

$$
\begin{aligned}
& H_{r}\left(\nabla \nabla u, \gamma_{1}(u), \gamma_{0}(u)\right) \\
\equiv & -\int_{\Omega} \mathbf{M}_{L}: \nabla \nabla u d x+r \int_{\Gamma_{1}}\left|\gamma_{1}(u)\right| d s+r\left\|\gamma_{0}(u)\right\|_{\gamma_{0}\left(W^{2,1}\right)} \\
& +\int_{\Gamma_{0}} I_{\left\{\gamma_{1}(u)=0\right\}}\left(\gamma_{1}(u)\right) d s+\int_{\Gamma_{0}} I_{\left\{\gamma_{0}(u)=0\right\}}\left(\gamma_{0}(u)\right) d s+\int_{\Omega} j(x, \nabla \nabla u) d x
\end{aligned}
$$

if $u \in W^{2,1}(\Omega)$, and $H_{r}\left(\nabla \nabla u, \gamma_{1}(u), \gamma_{0}(u)\right)=+\infty$ otherwise (where $\|\cdot\|_{\gamma_{0}\left(W^{2,1}\right)}=\|\cdot\|_{0}$, see $\left.(2.14)\right)$. Because of the equalities

$$
\begin{aligned}
\lambda \int_{\Omega} f u d x= & \int_{\Omega} \mathbf{M}_{L}: \nabla \nabla u d x-\int_{\operatorname{Fr} \Omega} \beta_{1}\left(\mathbf{M}_{L}\right) \gamma_{1}(u) d s \\
& +\left\langle\beta_{0}\left(\mathbf{M}_{L}\right) ; \gamma_{0}(u)\right\rangle_{\left(\gamma_{0}\left(W^{2,1}\right)\right)^{*} \times \gamma_{0}\left(W^{2,1}\right)} \\
\lambda \int_{\Gamma_{1}} g_{1} \gamma_{1}(u) d s= & \int_{\Gamma_{1}} \beta_{1}\left(\mathbf{M}_{L}\right) \gamma_{1}(u) d s \\
\lambda \int_{\Gamma_{1}} g_{0} \gamma_{0}(u) d s= & \int_{\Gamma_{1}} \beta_{0}\left(\mathbf{M}_{L}\right) \gamma_{0}(u) d s
\end{aligned}
$$

we obtain

$$
\begin{aligned}
& H_{r}\left(\nabla \nabla u, \gamma_{1}(u), \gamma_{0}(u)\right)=-\lambda \int_{\Omega} f u d x-\lambda \int_{\Gamma_{1}} g_{1} \gamma_{1}(u) d s \\
+ & r \int_{\Gamma_{1}}\left|\gamma_{1}(u)\right| d s+\lambda \int_{\Gamma_{1}} g_{0} \gamma_{0}(u) d s+r\left\|\gamma_{0}(u)\right\|_{\gamma_{0}\left(W^{2,1}\right)}+\int_{\Omega} j(x, \nabla \nabla u) d x
\end{aligned}
$$

if $u \in W^{2,1}(\Omega), \gamma_{1}(u)=0$ on $\Gamma_{0}, \gamma_{0}(u)=0$ on $\Gamma_{0}$, and $H_{r}\left(\nabla \nabla u, \gamma_{1}(u)\right.$, $\left.\gamma_{0}(u)\right)=+\infty$ otherwise (cf. (2.17), (4.21), Assumption 4 and [14, Chap. 3, (2.33)]). The functional $H_{r}$ describe the primal displacement problem for plates, with relaxed boundary conditions on $\Gamma_{1}$.

We define a linear space by

$$
\begin{array}{r}
A(\Omega) \equiv\left\{\left(\boldsymbol{\varphi}^{*}, \psi^{*}\right) \in W^{2}(\Omega, \operatorname{div} \operatorname{div}) \times\left(\gamma_{0}\left(W^{2,1}(\Omega)\right)\right)^{*} \mid\right. \\
\exists \psi_{1}^{*} \in C(\operatorname{Fr} \Omega), \exists \boldsymbol{\varphi}_{1}^{*} \in C_{\operatorname{div} \operatorname{div}}^{1}\left(\bar{\Omega}, \mathbf{E}_{s}^{2}\right), \exists \boldsymbol{\varphi}_{s}^{*} \in W_{\operatorname{div}}^{2}(\Omega, \operatorname{div} \operatorname{div}), \\
\left.\boldsymbol{\varphi}^{*}=\boldsymbol{\varphi}_{1}^{*}+\boldsymbol{\varphi}_{s}^{*} \text { and } \psi^{*}=\psi_{1}^{*}+\beta_{0}\left(\boldsymbol{\varphi}_{s}^{*}\right)\right\}
\end{array}
$$

(see (2.12)). Moreover, a net $\left\{u_{\delta}\right\}_{\delta \in D} \subset H B(\Omega)$ converges to $\widehat{u} \in H B(\Omega)$ 
in the topology $\sigma(\mathcal{Z}(H B(\Omega)) ; A(\Omega))$ if and only if

$$
\begin{aligned}
\int_{\Omega} \varphi^{*}: \nabla \nabla\left(u_{\delta}-\widehat{u}\right)- & \int_{\operatorname{Fr} \Omega} \beta_{1}\left(\varphi^{*}\right) \gamma_{1}\left(u_{\delta}-\widehat{u}\right) d s \\
& +\left\langle\psi^{*} ; \gamma_{0}\left(u_{\delta}-\widehat{u}\right)\right\rangle_{\left(\gamma_{0}\left(W^{2,1}\right)\right)^{*} \times \gamma_{0}\left(W^{2,1}\right)} \rightarrow 0
\end{aligned}
$$

for every $\left(\varphi^{*}, \psi^{*}\right) \in A(\Omega)$. Because of the duality between $\mathcal{Z}(H B(\Omega))$ and $A(\Omega)$ we obtain the dual functional $H_{r}^{*}: A(\Omega) \rightarrow \mathbb{R} \cup\{+\infty\}$ by setting

$$
\begin{aligned}
& H_{r}^{*}\left(\varphi^{*}, \psi^{*}\right)=\sup \left\{\int_{\Omega} \varphi^{*}: \nabla \nabla u d x-\int_{\operatorname{Fr} \Omega} \beta_{1}\left(\varphi^{*}\right) \gamma_{1}(u) d s\right. \\
& \left.+\left\langle\psi^{*} ; \gamma_{0}(u)\right\rangle_{\left(\gamma_{0}\left(W^{2,1}\right)\right)^{*} \times \gamma_{0}\left(W^{2,1}\right)}-H_{r}\left(\nabla \nabla u, \gamma_{1}(u), \gamma_{0}(u)\right) \mid u \in W^{2,1}(\Omega)\right\}
\end{aligned}
$$

for $\left(\varphi^{*}, \psi^{*}\right) \in A(\Omega)$. The bidual functional $H_{r}^{* *}: \mathcal{Z}(H B(\Omega)) \rightarrow \mathbb{R} \cup\{+\infty\}$ is defined by

$$
\begin{aligned}
& H_{r}^{* *}\left(\nabla \nabla u, \gamma_{1}(u), \gamma_{0}(u)\right)=\sup \left\{\int_{\Omega} \boldsymbol{\varphi}^{*}: \nabla \nabla u-\int_{\operatorname{Fr} \Omega} \beta_{1}\left(\varphi^{*}\right) \gamma_{1}(u) d s\right. \\
& \left.+\left\langle\psi^{*} ; \gamma_{0}(u)\right\rangle_{\left(\gamma_{0}\left(W^{2,1}\right)\right)^{*} \times \gamma_{0}\left(W^{2,1}\right)}-H_{r}^{*}\left(\boldsymbol{\varphi}^{*}, \psi^{*}\right) \mid\left(\boldsymbol{\varphi}^{*}, \psi^{*}\right) \in A(\Omega)\right\}
\end{aligned}
$$

for $u \in H B(\Omega)$. The extension $\widetilde{\mathcal{Z}}(H B(\Omega))$ of $\mathcal{Z}(H B(\Omega))$ is given by

$$
\begin{aligned}
& \widetilde{\mathcal{Z}}(H B(\Omega))=\left\{\left(\mathbf{z}, \gamma_{1}(u), w\right) \in \operatorname{span}\left[\nabla \nabla(H B(\Omega)), L^{1}\left(\Omega, \mathbf{E}_{s}^{2}\right)\right]\right. \\
& \times L^{1}(\operatorname{Fr} \Omega) \times \gamma_{0}\left(W^{2,1}(\Omega)\right) \mid \\
& \exists \widehat{\mathbf{z}} \in L^{1}\left(\Omega, \mathbf{E}_{s}^{2}\right), \exists \widehat{w} \in \gamma_{0}\left(W^{2,1}(\Omega)\right), \quad \exists \widetilde{u} \in H B(\Omega), \\
&\left.\mathbf{z}=\widehat{\mathbf{z}} d x+\nabla \nabla \widetilde{u}, \gamma_{1}(u)=\gamma_{1}(\widetilde{u}) \text { and } w=\widehat{w}+\gamma_{0}(\widetilde{u})\right\} .
\end{aligned}
$$

The bilinear pairing between $\widetilde{\mathcal{Z}}(H B(\Omega))$ and $A(\Omega)$ is given by

$$
\begin{aligned}
\left\langle\left(\mathbf{z}, \gamma_{1}(u), w\right) ;\left(\boldsymbol{\varphi}^{*}, \psi^{*}\right)\right\rangle_{1} \equiv & \int_{\Omega} \boldsymbol{\varphi}^{*}: \mathbf{z}-\int_{\operatorname{Fr} \Omega} \beta_{1}\left(\boldsymbol{\varphi}^{*}\right) \gamma_{1}(u) d s \\
& +\left\langle\psi^{*} ; w\right\rangle_{\left(\gamma_{0}\left(W^{2,1}\right)\right)^{*} \times \gamma_{0}\left(W^{2,1}\right)}
\end{aligned}
$$

for $\left(\mathbf{z}, \gamma_{1}(u), w\right) \in \widetilde{\mathcal{Z}}(H B(\Omega))$ and $\left(\varphi^{*}, \psi^{*}\right) \in A(\Omega)$. The extension $\widetilde{H}_{r}$ of $H_{r}$ onto the space $\widetilde{\mathcal{Z}}(H B(\Omega))$ is given by

$$
\begin{aligned}
\widetilde{H}_{r}\left(\mathbf{z}, \gamma_{1}(u), w\right) \\
=-\int_{\Omega} \mathbf{M}_{L}: \mathbf{z} d x+\int_{\operatorname{Fr} \Omega} \beta_{1}\left(\mathbf{M}_{L}\right) \gamma_{1}(u) d s \\
\quad-\left\langle\beta_{0}\left(\mathbf{M}_{L}\right) ; w\right\rangle_{\left(\gamma_{0}\left(W^{2,1}\right)\right)^{*} \times \gamma_{0}\left(W^{2,1}\right)}-\int_{\Gamma_{1}} \beta_{1}\left(\mathbf{M}_{L}\right) \gamma_{1}(u) d s
\end{aligned}
$$




$$
\begin{aligned}
& +\int_{\Gamma_{1}} \beta_{0}\left(\mathbf{M}_{L}\right) w d s+r \int_{\Gamma_{1}}\left|\gamma_{1}(u)\right| d s+r\|w\|_{\gamma_{0}\left(W^{2,1}\right)} \\
& +\int_{\Gamma_{0}} I_{\left\{\gamma_{1}(u)=0\right\}}\left(\gamma_{1}(u)\right) d s+\int_{\Gamma_{0}} I_{\{w=0\}}(w) d s+\int_{\Omega} j(x, \mathbf{z}) d x
\end{aligned}
$$

if $\mathbf{z}=\widehat{\mathbf{z}} d x+\nabla \nabla \widetilde{u}, \gamma_{1}(u)=\gamma_{1}(\widetilde{u})$ and $w=\widehat{w}+\gamma_{0}(\widetilde{u})$, with $\widehat{\mathbf{z}} \in L^{1}\left(\Omega, \mathbf{E}_{s}^{2}\right)$, $\widehat{w} \in \gamma_{0}\left(W^{2,1}(\Omega)\right), \widetilde{u} \in W^{2,1}(\Omega)$, and $\widetilde{H}_{r}\left(\mathbf{z}, \gamma_{1}(u), w\right)=+\infty$ otherwise. By the duality between $\widetilde{\mathcal{Z}}(H B(\Omega))$ and $A(\Omega)$ we define a functional $\widetilde{H}_{r}^{*}$ on the linear space $A(\Omega)$ by

$$
\begin{array}{r}
\widetilde{H}_{r}^{*}\left(\varphi^{*}, \psi^{*}\right)=\sup \left\{\left\langle\left(\mathbf{z}, \gamma_{1}(u), w\right) ;\left(\varphi^{*}, \psi^{*}\right)\right\rangle_{1}-\widetilde{H}_{r}\left(\mathbf{z}, \gamma_{1}(u), w\right) \mid\right. \\
\left.\left(\mathbf{z}, \gamma_{1}(u), w\right) \in \widetilde{\mathcal{Z}}(H B(\Omega))\right\}
\end{array}
$$

for $\left(\varphi^{*}, \psi^{*}\right) \in A(\Omega)$. The bidual functional $\widetilde{H}_{r}^{* *}: \widetilde{\mathcal{Z}}(H B(\Omega)) \rightarrow \mathbb{R} \cup\{+\infty\}$ is defined by

$$
\begin{array}{r}
\widetilde{H}_{r}^{* *}\left(\mathbf{z}, \gamma_{1}(u), w\right)=\sup \left\{\left\langle\left(\mathbf{z}, \gamma_{1}(u), w\right) ;\left(\boldsymbol{\varphi}^{*}, \psi^{*}\right)\right\rangle_{1}-\widetilde{H}_{r}^{*} \varphi^{*}, \psi^{*}\right) \mid \\
\left.\left(\boldsymbol{\varphi}^{*}, \psi^{*}\right) \in A(\Omega)\right\}
\end{array}
$$

for $\left(\mathbf{z}, \gamma_{1}(u), w\right) \in \widetilde{\mathcal{Z}}(H B(\Omega))$.

Proposition 11. The explicit form of $\widetilde{H}_{r}^{*}$ is

$$
\begin{aligned}
\widetilde{H}_{r}^{*}\left(\boldsymbol{\varphi}^{*}, \psi^{*}\right)= & \int_{\Gamma_{1}} I_{B_{\mathbb{R}}(0, r)}\left(-\beta_{1}\left(\boldsymbol{\varphi}^{*}\right)\right) d s+I_{B_{\left(\gamma_{0}\left(W^{2,1}\right)\right)^{*}}(0, r)}\left(\psi_{\mid \operatorname{int} \Gamma_{1}}^{*}\right) \\
& +\int_{\Omega} j^{*}\left(x,\left(\boldsymbol{\varphi}^{*}+\mathbf{M}_{L}\right)\right) d x
\end{aligned}
$$

for $\left(\varphi^{*}, \psi^{*}\right) \in A(\Omega)$. Here $\psi_{\mid \text {int } \Gamma_{1}}^{*}$ denotes the functional $\widetilde{\psi}^{*} \in\left(\gamma_{0}\left(W^{2,1}(\Omega)\right)\right)^{*}$ such that $\widetilde{\psi}^{*}=\psi^{*}$ on $\operatorname{int} \Gamma_{1}$ and $\widetilde{\psi}^{*}=0$ on $\operatorname{cl} \Gamma_{0}$ (see (4.21)). If we extend $\widetilde{H}_{r}^{*}$ onto the space $W^{2}(\Omega$, div div $) \times\left(\gamma_{0}\left(W^{2,1}\right)\right)^{*}$ by $(4.35)$, then $\widetilde{H}_{r}^{*}$ is given by $(4.37)$ for every $\left(\varphi^{*}, \psi^{*}\right) \in W^{2}(\Omega, \operatorname{div} \operatorname{div}) \times\left(\gamma_{0}\left(W^{2,1}\right)\right)^{*}$.

Proof. By Theorem 3A of [13] we obtain

$$
\begin{array}{r}
\tilde{H}_{r}^{*}\left(\boldsymbol{\varphi}^{*}, \psi^{*}\right)=\sup \left\{\int_{\Omega} \varphi^{*}: \mathbf{z} d x-\int_{\operatorname{Fr} \Omega} \beta_{1}\left(\boldsymbol{\varphi}^{*}\right) \gamma_{1}(u) d s\right. \\
+\left\langle\psi^{*} ; w\right\rangle_{\left(\gamma_{0}\left(W^{2,1}\right)\right)^{*} \times \gamma_{0}\left(W^{2,1}\right)}+\int_{\Omega} \mathbf{M}_{L}: \mathbf{z} d x-r \int_{\Gamma_{1}}\left|\gamma_{1}(u)\right| d s \\
-r\|w\|_{\gamma_{0}\left(W^{2,1}\right)}-\int_{\Gamma_{0}} I_{\left\{\gamma_{1}(u)=0\right\}}\left(\gamma_{1}(u)\right) d s-\int_{\Gamma_{0}} I_{\{w=0\}}(w) d s-\int_{\Omega} j(x, \mathbf{z}) d x \mid \\
\left.\mathbf{z} \in L^{1}\left(\Omega, \mathbf{E}_{s}^{2}\right), u \in W^{2,1}(\Omega), w \in \gamma_{0}\left(W^{2,1}(\Omega)\right)\right\}
\end{array}
$$




$$
\begin{aligned}
& =\sup \left\{\int_{\Omega}\left(\boldsymbol{\varphi}^{*}+\mathbf{M}_{L}\right): \mathbf{z} d x-\int_{\Omega} j(x, \mathbf{z}) d x \mid \mathbf{z} \in L^{1}\left(\Omega, \mathbf{E}_{s}^{2}\right)\right\} \\
& +\sup \left\{-\int_{\operatorname{Fr} \Omega} \beta_{1}\left(\varphi^{*}\right) \gamma_{1}(u) d s-r \int_{\Gamma_{1}}\left|\gamma_{1}(u)\right| d s\right. \\
& \left.\quad-\int_{\Gamma_{0}} I_{\left\{\gamma_{1}(u)=0\right\}}\left(\gamma_{1}(u)\right) d s \mid u \in W^{2,1}(\Omega)\right\} \\
& +\sup \left\{\left\langle\psi^{*} ; w\right\rangle_{\left(\gamma_{0}\left(W^{2,1}\right)\right)^{*} \times \gamma_{0}\left(W^{2,1}\right)}-r\|w\|_{\gamma_{0}\left(W^{2,1}\right)} \mid\right. \\
& \left.w \in \gamma_{0}\left(W^{2,1}(\Omega)\right) \text { and } w=0 \text { on } \operatorname{cl} \Gamma_{0}\right\}
\end{aligned}
$$

for every $\left(\varphi^{*}, \psi^{*}\right) \in A(\Omega)$, since $\gamma_{1}$ is a surjection on $L^{1}(\operatorname{Fr} \Omega)$ (cf. [8]). Then we obtain $(4.37)$.

Define $\widetilde{H}_{r}^{* \#}: \widetilde{\mathcal{Z}}(H B) \rightarrow \mathbb{R} \cup\{+\infty\}$ by

$$
\begin{array}{r}
\widetilde{H}_{r}^{* \#}\left(\mathbf{z}, \gamma_{1}(u), w\right)=\sup \left\{\left\langle\left(\mathbf{z}, \gamma_{1}(u), w\right) ;\left(\varphi^{*}, \psi^{*}\right)\right\rangle_{1}-\widetilde{H}_{r}^{*}\left(\boldsymbol{\varphi}^{*}, \psi^{*}\right) \mid\right. \\
\left.\left(\boldsymbol{\varphi}^{*}, \psi^{*}\right) \in C_{\operatorname{div} \operatorname{div}}^{1}\left(\bar{\Omega}, \mathbf{E}_{s}^{2}\right) \times C(\operatorname{Fr} \Omega)\right\}
\end{array}
$$

for $\left(\mathbf{z}, \gamma_{1}(u), w\right) \in \widetilde{\mathcal{Z}}(H B(\Omega))$

Proposition 12. The explicit form of $\widetilde{H}_{r}^{* \#}$ is

$$
\begin{aligned}
\widetilde{H}_{r}^{* \#}\left(\nabla \nabla u, \gamma_{1}(u), \gamma_{0}(u)\right)=-\lambda \int_{\Omega} f u d x-\lambda \int_{\Gamma_{1}} g_{1} \gamma_{1}(u) d s \\
+r \int_{\Gamma_{1}}\left|\gamma_{1}(u)\right| d s+\lambda \int_{\Gamma_{1}} g_{0} \gamma_{0}(u) d s+r\left\|\gamma_{0}(u)\right\|_{\gamma_{0}\left(W^{2,1}\right)} \\
+\int_{\Gamma_{0}} j_{\infty}\left(x,-\boldsymbol{\nu} \otimes \gamma_{1}(u)\right) d s+\int_{\Gamma_{0}} I_{\left\{\gamma_{0}(u)=0\right\}}\left(\gamma_{0}(u)\right) d s \\
+\int_{\Omega} j\left(x,(\nabla \nabla u)_{a}\right) d x+\int_{\Omega} j_{\infty}\left(x, \frac{d(\nabla \nabla u)_{s}}{d\left|(\nabla \nabla u)_{s}\right|}\right) d\left|(\nabla \nabla u)_{s}\right|
\end{aligned}
$$

for every $u \in H B(\Omega)$.

Proof. The field $\mathbf{M}_{L}$ is a solution of $\left(P_{\lambda, r}^{*}\right)$, i.e. $\left\lceil P_{\lambda, r}^{*}\right\rceil\left(\mathbf{M}_{L}\right)=\sup \left(P_{\lambda, r}^{*}\right)$ (cf. $(4.21))$. Thus $\widetilde{H}_{r}^{*}(0,0)<\infty$. Moreover, $C_{\operatorname{div} \operatorname{div}}^{1}\left(\bar{\Omega}, \mathbf{E}_{s}^{2}\right)$ and $C(\operatorname{Fr} \Omega)$ are PCU-stable, so by [5] and by Theorem $3 \mathrm{~A}$ of [13] we get

$$
\widetilde{H}_{r}^{* \#}\left(\nabla \nabla u, \gamma_{1}(u), \gamma_{0}(u)\right)=\sup \left\{\int_{\Omega} \varphi^{*}: \nabla \nabla u-\int_{\operatorname{Fr} \Omega} \beta_{1}\left(\varphi^{*}\right) \gamma_{1}(u) d s\right.
$$




$$
\begin{aligned}
& +\left\langle\psi^{*} ; \gamma_{0}(u)\right\rangle_{\left(\gamma_{0}\left(W^{2,1}\right)\right)^{*} \times \gamma_{0}\left(W^{2,1}\right)} \\
& -\int_{\Gamma_{1}} I_{B_{\mathbb{R}}(0, r)}\left(-\beta_{1}\left(\boldsymbol{\varphi}^{*}\right)\right) d s-I_{B_{\left(\gamma_{0}\left(W^{2,1}\right)\right)^{*}}(0, r)}\left(\psi_{\mid \operatorname{int} \Gamma_{1}}^{*}\right) \\
& \left.-\int_{\Omega} j^{*}\left(x,\left(\boldsymbol{\varphi}^{*}+\mathbf{M}_{L}\right)\right) d x \mid\left(\boldsymbol{\varphi}^{*}, \psi^{*}\right) \in C_{\operatorname{div} \operatorname{div}}^{1}\left(\bar{\Omega}, \mathbf{E}_{s}^{2}\right) \times C(\operatorname{Fr} \Omega)\right\} \\
& =\sup \left\{\int_{\Omega}\left[\left(\boldsymbol{\varphi}^{*}+\mathbf{M}_{L}\right):(\nabla \nabla u)_{a}-j^{*}\left(x,\left(\boldsymbol{\varphi}^{*}+\mathbf{M}_{L}\right)\right)\right] d x\right. \\
& +\int_{\Omega}\left[\left(\boldsymbol{\varphi}^{*}+\mathbf{M}_{L}\right): \frac{d(\nabla \nabla u)_{s}}{d\left|(\nabla \nabla u)_{s}\right|}-j_{\infty}^{*}\left(x,\left(\boldsymbol{\varphi}^{*}+\mathbf{M}_{L}\right)\right)\right] d\left|(\nabla \nabla u)_{s}\right| \\
& +\int_{\Gamma_{1}}\left[\left(-\beta_{1}\left(\boldsymbol{\varphi}^{*}\right)\right) \gamma_{1}(u)-I_{B_{\mathbb{R}}(0, r)}\left(-\beta_{1}\left(\boldsymbol{\varphi}^{*}\right)\right)\right] d s \\
& +\int_{\Gamma_{0}}\left[\left(\boldsymbol{\varphi}^{*}+\mathbf{M}_{L}\right):\left(-\boldsymbol{\nu} \otimes \boldsymbol{\nu} \gamma_{1}(u)\right)-j_{\infty}^{*}\left(x,\left(\boldsymbol{\varphi}^{*}+\mathbf{M}_{L}\right)\right)\right] d s \mid \\
& \left.+\boldsymbol{\varphi}^{*} \in C_{\operatorname{div} \operatorname{div}}^{1}\left(\bar{\Omega}, \mathbf{E}_{s}^{2}\right)\right\} \\
& +\int_{\Omega} \mathbf{M}_{L}:(\nabla \nabla u)+\int_{\Gamma_{0}}\left\{\beta_{1}\left(\mathbf{M}_{L}\right) \gamma_{1}(u) d s\right.
\end{aligned}
$$

for every $u \in H B(\Omega)$, which is (4.40) (cf. (4.24), (4.25) and (4.26)). Here $\nabla \nabla u=(\nabla \nabla u)_{a}+(\nabla \nabla u)_{s}$ is the Lebesgue decomposition of $\nabla \nabla u$ into absolutely continuous and singular parts with respect to $d x$. In the above calculations we have used the equality $j_{\infty}^{*}\left(x, \varphi^{*}\right)=I_{\mathcal{K}_{p}(x)}\left(\varphi^{*}\right)$, which holds for every $\varphi^{*} \in \mathbf{E}_{s}^{2}$ and $x \in \bar{\Omega}$.

Definition 2. We say that $H_{r}$ is coercive if

$$
H_{r}\left(\nabla \nabla u_{m}, \gamma_{1}\left(u_{m}\right), \gamma_{0}\left(u_{m}\right)\right) \rightarrow+\infty
$$

for every sequence $\left\{u_{m}\right\}_{m \in \mathbb{N}} \subset H B(\Omega)$ such that $\left\|u_{m}\right\|_{H B(\Omega)} \rightarrow \infty$.

Assumption 5. Let $H_{r}$ be a coercive function. Moreover, let $\widetilde{H}_{r}^{* \#}$ be the largest minorant that is less than $H_{r}$ and l.s.c. in the weak $H B$ topology.

Definition 3. We say that a net $\left\{u_{\delta}\right\}_{\delta \in D} \subset H B(\Omega)$ converges to $u_{0} \in$ $H B(\Omega)$ in the topology (4.43)-(4.44) if

$$
\left\langle\left(\nabla \nabla\left(u_{\delta}-u_{0}\right), \gamma_{1}\left(u_{\delta}-u_{0}\right), \gamma_{0}\left(u_{\delta}-u_{0}\right)\right) ;\left(\varphi^{*}, \beta_{0}\left(\varphi^{*}\right)\right)\right\rangle_{1} \rightarrow 0
$$


for every $\varphi^{*} \in W^{2}(\Omega, \operatorname{div} \operatorname{div})$ and

$$
\left\langle\left(\nabla \nabla\left(u_{\delta}-u_{0}\right), \gamma_{1}\left(u_{\delta}-u_{0}\right), \gamma_{0}\left(u_{\delta}-u_{0}\right)\right) ;\left(\widetilde{\varphi}^{*}, \psi^{*}\right)\right\rangle_{1} \rightarrow 0
$$

for every $\left(\widetilde{\varphi}^{*}, \psi^{*}\right) \in C_{\operatorname{div} \operatorname{div}}^{1}\left(\bar{\Omega}, \mathbf{E}_{s}^{2}\right) \times C(\operatorname{Fr} \Omega)$.

LEMMA 13. Every closed ball $\mathrm{cl}_{\|\cdot\|_{H B}}(B(0, \widehat{r}))($ in $\mathcal{Z}(H B(\Omega)))$ is compact in the topology (4.43)-(4.44).

Proof. Let $\left\{u_{\delta}\right\}_{\delta \in D} \subset H B(\Omega)$ be a net in $B_{H B}(0, \widehat{r})$. Then, by Theorem 8 , there exists a finer net $\left\{u_{\delta_{\beta}}\right\}_{\beta \in B} \subset H B(\Omega)$ and $\widetilde{u} \in \operatorname{cl}_{\|\cdot\|} B_{H B}(0, \widehat{r})$ such that $\left\{u_{\delta_{\beta}}\right\}$ converges to $\widetilde{u}$ in the sense of (4.43). Moreover, by Theorem 9 , there exists a net $\left\{u_{\delta_{\beta_{\gamma}}}\right\}$ (finer than $\left\{u_{\delta_{\beta}}\right\}_{\beta \in B}$ ) and $\widehat{u} \in \operatorname{cl}_{\|\cdot\|} B_{H B}(0, \widehat{r})$ such that $\left\{u_{\delta_{\beta_{\gamma}}}\right\}$ converges to $\widehat{u}$ in the sense of (4.44). Therefore $\left\{u_{\delta_{\beta_{\gamma}}}\right\}$ converges to $\widehat{u} \in \mathrm{cl}_{\|\cdot\|} B_{H B}(0, \widehat{r})$ in the topology $(4.43)-(4.44)$.

Corollary 14. Every closed ball $\mathrm{cl}_{\|\cdot\|_{H B}}(B(0, \widehat{r}))($ in $\mathcal{Z}(H B(\Omega)))$ is compact in the topology $\sigma(\mathcal{Z}(H B(\Omega)) ; A(\Omega))$ (cf. (4.29)). Moreover, the topologies (4.43)-(4.44) and $\sigma(\mathcal{Z}(H B(\Omega)) ; A(\Omega))$ are equivalent on each such ball.

Proof. The Hausdorff topology $\sigma(\mathcal{Z}(H B(\Omega)) ; A(\Omega))$ is weaker than the topology (4.43)-(4.44). Then, by [11, Corollary 3.1.14] and Lemma 13, we obtain the equivalence of the two topologies on $\mathrm{cl}_{\|\cdot\|_{H B}}(B(0, \widehat{r}))$.

Proposition 15. The space $\left[\operatorname{cl}_{\|\cdot\|_{H B}}(B(0, \widehat{r}))\right.$, weak* topology $]$ is homeomorphic to the space $\left[\mathcal{Z}\left(\operatorname{cl}_{\|\cdot\|_{H B}}(B(0, \widehat{r}))\right), \sigma(\mathcal{Z}(H B(\Omega)) ; A(\Omega))\right]$.

Proof. The Hausdorff topology $\sigma\left(\mathcal{Z}(H B) ; W^{2}(\Omega\right.$, divdiv $\left.)\right)$ is weaker than (4.43)-(4.44). Then (by [11, Corollary 3.1.14], Corollary 14 and Theorem 10) we obtain the equivalence of these topologies on $\mathrm{cl}_{\|\cdot\|_{H B}}(B(0, \widehat{r}))$.

Lemma 16. For every $\left(\boldsymbol{\varphi}^{*}, \psi^{*}\right) \in A(\Omega)$ we have $\widetilde{H}_{r}^{*}\left(\boldsymbol{\varphi}^{*}, \psi^{*}\right) \geq H_{r}^{*}\left(\boldsymbol{\varphi}^{*}, \psi^{*}\right)$. Moreover, $\widetilde{H}_{r}^{* *}(\widehat{\mathbf{z}}) \leq H_{r}^{* *}(\widehat{\mathbf{z}})$ for every $\widehat{\mathbf{z}} \in \mathcal{Z}(H B(\Omega))$.

Proposition 17. Under Assumption $5, \widetilde{H}_{r}^{* \#}(\widehat{\mathbf{z}})=\widetilde{H}_{r}^{* *}(\widehat{\mathbf{z}})=H_{r}^{* *}(\widehat{\mathbf{z}})$ for every $\widehat{\mathbf{z}} \in \mathcal{Z}(H B(\Omega))$.

Proof. In the definition of $\widetilde{H}_{r}^{* *}$ we take the supremum over a larger domain, so $\widetilde{H}_{r}^{* \#} \leq \widetilde{H}_{r}^{* *}$. By Lemma $16, \widetilde{H}_{r}^{* \#} \leq \widetilde{H}_{r}^{* *} \leq H_{r}^{* *}$. Because of (4.30) and (4.31), $H_{r}^{* *}$ is the l.s.c. regularization of $H_{r}$ in $\sigma(\mathcal{Z}(H B(\Omega)) ; A(\Omega))$. By Assumption 5 and Proposition 15 we get $\widetilde{H}_{r}^{* \#}=H_{r}^{* *}$.

Lemma 18. Let Assumption 5 hold. For every $u \in W^{2,1}(\Omega)$ such that $\gamma_{1}(u)_{\mid \Gamma_{0}}=0$, we have $\widetilde{H}_{r}^{* *}\left(\nabla \nabla u, \gamma_{1}(u), \gamma_{0}(u)\right)=H_{r}\left(\nabla \nabla u, \gamma_{1}(u), \gamma_{0}(u)\right)$.

Proof. By Lemma 16, $\widetilde{H}_{r}^{* *} \leq H_{r}^{* *} \leq H_{r}$. Then, by (4.40) and Proposition 17 , we obtain the conclusion of the lemma. 
Lemma 19. Let $\varphi_{s}^{*} \in W_{\text {div }}^{2}(\Omega, \operatorname{div} \operatorname{div})$. Then

$$
H_{r}^{*}\left(\boldsymbol{\varphi}^{*}, \psi^{*}\right)=H_{r}^{*}\left(\boldsymbol{\varphi}^{*}+\boldsymbol{\varphi}_{s}^{*}, \psi^{*}+\beta_{0}\left(\boldsymbol{\varphi}_{s}^{*}\right)\right),
$$

for every $\varphi^{*} \in C_{\operatorname{div} \operatorname{div}}^{1}\left(\bar{\Omega}, \mathbf{E}_{s}^{2}\right)$ and $\psi^{*} \in C(\operatorname{Fr} \Omega, \mathbb{R})(c f .(2.12))$.

Proof. By (4.30) and (2.17) we obtain

$$
\begin{aligned}
H_{r}^{*}\left(\boldsymbol{\varphi}^{*}, \psi^{*}\right) & \sup \left\{\int_{\Omega} u\left(\operatorname{div} \operatorname{div} \boldsymbol{\varphi}^{*}\right) d x\right. \\
& -\left\langle\left(\beta_{0}\left(\boldsymbol{\varphi}^{*}\right)-\psi^{*}\right) ; \gamma_{0}(u)\right\rangle_{\left(\gamma_{0}\left(W^{2,1}\right)\right)^{*} \times \gamma_{0}\left(W^{2,1}\right)} \\
& \left.-H_{r}\left(\nabla \nabla u, \gamma_{1}(u), \gamma_{0}(u)\right) \mid u \in W^{2,1}(\Omega)\right\} \\
= & \sup \left\{\int_{\Omega} u\left(\operatorname{div} \operatorname{div}\left(\boldsymbol{\varphi}^{*}+\boldsymbol{\varphi}_{s}^{*}\right)\right) d x\right. \\
& +\left\langle\left(\psi^{*}+\beta_{0}\left(\boldsymbol{\varphi}_{s}^{*}\right)-\beta_{0}\left(\boldsymbol{\varphi}^{*}+\boldsymbol{\varphi}_{s}^{*}\right)\right) ; \gamma_{0}(u)\right\rangle_{\left(\gamma_{0}\left(W^{2,1}\right)\right)^{*} \times \gamma_{0}\left(W^{2,1}\right)} \\
& \left.-H_{r}\left(\nabla \nabla u, \gamma_{1}(u), \gamma_{0}(u)\right) \mid u \in W^{2,1}(\Omega)\right\} \\
= & H_{r}^{*}\left(\boldsymbol{\varphi}^{*}+\boldsymbol{\varphi}_{s}^{*}, \psi^{*}+\beta_{0}\left(\boldsymbol{\varphi}_{s}^{*}\right)\right)
\end{aligned}
$$

for every $\left(\boldsymbol{\varphi}^{*}, \psi^{*}\right) \in C_{\operatorname{div} \operatorname{div}}^{1}\left(\bar{\Omega}, \mathbf{E}_{s}^{2}\right) \times C(\operatorname{Fr} \Omega)$.

Let $\operatorname{ker} \nabla \nabla(\cdot) \equiv\{u \in H B \mid \nabla \nabla u=0\}$. Then

$$
\operatorname{ker} \nabla \nabla(\cdot)=\left\{u \in W^{2,1} \mid u(z, y)=a z+b y+c \text { and } a, b, c \in \mathbb{R}\right\} .
$$

There exist continuous projections of the space $W^{2,1}(\Omega)$ on

$$
\begin{aligned}
\widetilde{P}_{I} & =\left\{u \in W^{2,1}(\Omega) \mid u(z, y)=c \text { and } c \in \mathbb{R}\right\}, \\
\widetilde{P}_{I I} & =\left\{u \in W^{2,1}(\Omega) \mid u(z, y)=a z \text { and } a \in \mathbb{R}\right\}, \\
\widetilde{P}_{I I I} & =\left\{u \in W^{2,1}(\Omega) \mid u(z, y)=b y \text { and } b \in \mathbb{R}\right\},
\end{aligned}
$$

given by

$$
\begin{aligned}
W^{2,1}(\Omega) \ni u & \mapsto P_{I}(u)=\frac{1}{\operatorname{meas} \Omega} \int_{\Omega} u(z, y) d z d y, \\
W^{2,1}(\Omega) \ni u \mapsto P_{I I}(u) & =\frac{z}{\operatorname{meas} \Omega}\left(\int_{\Omega} \frac{\partial u(z, y)}{\partial z} d z d y\right) .
\end{aligned}
$$

The projection $P_{I I I}$ of $W^{2,1}(\Omega)$ on $\widetilde{P}_{I I I}$ is defined similarly to (4.52). Hence there exists a decomposition $W^{2,1}(\Omega)=\operatorname{ker} \nabla \nabla(\cdot) \oplus\left[W^{2,1}(\Omega) / \operatorname{ker} \nabla \nabla(\cdot)\right]$, given by

$$
u=\left(P_{I}(u)+P_{I I}(u)+P_{I I I}(u)\right)+\left\{u-\left(P_{I}(u)+P_{I I}(u)+P_{I I I}(u)\right)\right\} .
$$


Lemma 20. The trace $\left(\beta_{0}, \beta_{1}\right): W_{\text {div }}^{2}(\Omega$, div div $) \rightarrow\left\{\left(\gamma_{0}(u), \gamma_{1}(u)\right) \in\right.$ $\left.\gamma_{0}\left(W^{2,1}(\Omega)\right) \times L^{1}(\operatorname{Fr} \Omega) \mid u \in\left[W^{2,1}(\Omega) / \operatorname{ker} \nabla \nabla(\cdot)\right]\right\}^{*}$ is a surjection.

Proof. The proof is similar to Step 2 of the proof of [14, Chap. 3, Proposition 2.11]. Let $\left(L_{0}, L_{1}\right) \in\left(\gamma_{0}\left(W^{2,1}(\Omega)\right)\right)^{*} \times L^{\infty}(\operatorname{Fr} \Omega), L_{0 \mid \gamma_{0}(\operatorname{ker} \nabla \nabla(\cdot))}=0$ and $L_{1 \mid \gamma_{1}(\operatorname{ker} \nabla \nabla(\cdot))}=0$. Then

$$
\begin{aligned}
W^{2,1}(\Omega) \ni u & \mapsto l(u) \\
& =\int_{\operatorname{Fr} \Omega} L_{1} \gamma_{1}(u) d s-\left\langle L_{0} ; \gamma_{0}(u)\right\rangle_{\left(\gamma_{0}\left(W^{2,1}\right)\right)^{*} \times \gamma_{0}\left(W^{2,1}\right)}
\end{aligned}
$$

is a continuous, linear functional on $W^{2,1}(\Omega)$ such that $l(u)=0$ if $u \in$ $\operatorname{ker} \nabla \nabla(\cdot)$. Because of [14, Chap. 3, (2.82)] the operator $u \mapsto \nabla \nabla u$ is an isomorphism of $W^{2,1}(\Omega) / \operatorname{ker} \nabla \nabla(\cdot)$ into $L^{1}\left(\Omega, \mathbf{E}_{s}^{2}\right)$. By the Hahn-Banach theorem, there exists an extension of the form $l$, with the same norm, on the space $L^{1}\left(\Omega, \mathbf{E}_{s}^{2}\right)$. Then there exists $\mathbf{M} \in L^{\infty}\left(\Omega, \mathbf{E}_{s}^{2}\right)$ such that $l(u)=$ $\int_{\Omega} \mathbf{M}: \nabla \nabla u d x$ for all $u \in W^{2,1}(\Omega) / \operatorname{ker} \nabla \nabla(\cdot)$. If $\widetilde{u} \in C_{\mathrm{c}}^{\infty}(\Omega)$ then $l(\widetilde{u})=0$. Since $l(u)=\int_{\Omega} \mathbf{M}: \nabla \nabla u d x-\int_{\Omega} 0 u d x$ for all $u \in W^{2,1}(\Omega) / \operatorname{ker} \nabla \nabla(\cdot)$, we have $\operatorname{div}(\operatorname{div} \mathbf{M})=0$ in $\Omega$. Due to Step 1 of the proof of [14, Chap. 3, Proposition 2.11] we see that $L_{0}=\beta_{0}(\mathbf{M})$ and $L_{1}=\beta_{1}(\mathbf{M})$. Finally since $l$ is extended to a linear mapping with the same norm,

$$
\begin{aligned}
\|l\| & =\left\{\left\|L_{0}\right\|_{\left(\gamma_{0}\left(W^{2,1}\right)\right)^{*}}+\left\|L_{1}\right\|_{L^{\infty}}\right\}=\|\mathbf{M}\|_{L^{\infty}\left(\Omega, \mathbf{E}_{s}^{2}\right)} \\
& =\|\mathbf{M}\|_{W^{2}(\Omega, \text { div div })},
\end{aligned}
$$

and this completes the proof of surjectivity.

We say that a net $\left\{\left(\boldsymbol{\varphi}_{k}^{*}, \psi_{k}^{*}\right)\right\}_{k \in K} \subset A(\Omega)$ converges to $\left(\widehat{\boldsymbol{\varphi}}^{*}, \widehat{\psi}^{*}\right) \in A(\Omega)$ in the topology

$$
\sigma\left(A(\Omega) ; L^{\infty}\left(\Omega, \mathbf{E}_{s}^{2}\right) \times L^{\infty}\left(\Gamma_{1}, \mathbb{R}\right) \times \gamma_{0}\left(W^{2,1}(\Omega)\right)_{\mid \Gamma_{1}}\right)
$$

if

$$
\begin{aligned}
\int_{\Omega}\left(\boldsymbol{\varphi}_{k}^{*}-\widehat{\boldsymbol{\varphi}}_{k}^{*}\right): \mathbf{z} d x-\int_{\Gamma_{1}} \boldsymbol{\beta}_{1}\left(\boldsymbol{\varphi}_{k}^{*}-\widehat{\boldsymbol{\varphi}}_{k}^{*}\right) h d s \\
\quad+\left\langle\left(\psi_{k}^{*}-\widehat{\psi}_{k}^{*}\right) ; w\right\rangle\left(\gamma_{0}\left(W^{2,1}\right)\right)^{*} \times \gamma_{0}\left(W^{2,1}\right) \rightarrow 0
\end{aligned}
$$

for every $\mathbf{z} \in L^{\infty}\left(\Omega, \mathbf{E}_{s}^{2}\right), h \in L^{\infty}\left(\Gamma_{1}\right)$ and $w \in \gamma_{0}\left(W^{2,1}\right)$ with $w_{\mid \Gamma_{0}}=0$.

Lemma 21. Let $\widehat{f}: A(\Omega) \rightarrow \mathbb{R}$ be a linear functional, continuous in the topology (4.56), such that $\widehat{f}\left(\boldsymbol{\varphi}_{s}^{*}, \boldsymbol{\beta}_{0}\left(\boldsymbol{\varphi}_{s}^{*}\right)\right)=0$ for every $\boldsymbol{\varphi}_{s}^{*} \in W_{\text {div }}^{2}(\Omega$, div div) (cf. (2.12)). Then there exists $\widetilde{u} \in W^{2,1}(\Omega)$ such that $\gamma_{1}(\widetilde{u})=0=\gamma_{0}(\widetilde{u})$ on $\Gamma_{0}, \nabla \nabla \widetilde{u} \in L^{\infty}\left(\Omega, \mathbf{E}_{s}^{2}\right), \gamma_{1}(\widetilde{u}) \in L^{\infty}(\operatorname{Fr} \Omega)$ and

$$
\begin{aligned}
\widehat{f}\left(\boldsymbol{\varphi}^{*}, \psi^{*}\right)= & \int_{\Omega} \boldsymbol{\varphi}^{*}:(\nabla \nabla \widetilde{u}) d x-\int_{\operatorname{Fr} \Omega} \beta_{1}\left(\varphi^{*}\right) \gamma_{1}(\widetilde{u}) d s \\
& +\left\langle\psi^{*} ; \gamma_{0}(\widetilde{u})\right\rangle_{\left(\gamma_{0}\left(W^{2,1}\right)\right)^{*} \times \gamma_{0}\left(W^{2,1}\right)}
\end{aligned}
$$

for $\left(\varphi^{*}, \psi^{*}\right) \in A(\Omega)$. 
Proof. Step 1. The functional $\widehat{f}$ is continuous in the topology (4.56). So, by Theorem V.3.9 of [9], there exist $\widetilde{\varkappa} \in L^{\infty}\left(\Omega, \mathbf{E}_{s}^{2}\right)$ and $\widehat{u} \in W^{2,1}(\Omega)$ such that $\gamma_{1}(\widehat{u})=0=\gamma_{0}(\widehat{u})$ on $\Gamma_{0}, \gamma_{1}(\widehat{u}) \in L^{\infty}(\operatorname{Fr} \Omega)$ and

$$
\begin{aligned}
\widehat{f}\left(\varphi^{*}, \psi^{*}\right)= & \int_{\Omega} \boldsymbol{\varphi}^{*}: \widetilde{\varkappa} d x-\int_{\operatorname{Fr} \Omega} \beta_{1}\left(\varphi^{*}\right) \gamma_{1}(\widehat{u}) d s \\
& +\left\langle\psi^{*} ; \gamma_{0}(\widehat{u})\right\rangle_{\left(\gamma_{0}\left(W^{2,1}\right)\right)^{*} \times \gamma_{0}\left(W^{2,1}\right)}
\end{aligned}
$$

for all $\left(\varphi^{*}, \psi^{*}\right) \in A(\Omega)$. Indeed, the trace $\left(\gamma_{1}, \gamma_{0}\right): W^{2,1}(\Omega) \rightarrow L^{1}(\operatorname{Fr} \Omega) \times$ $\gamma_{0}\left(W^{2,1}(\Omega)\right)$ is a surjection and $L^{\infty}(\operatorname{Fr} \Omega) \subset L^{1}(\operatorname{Fr} \Omega)$, because $\Omega$ is a bounded domain of class $C^{2}$ (see [8] and [14, Chap. 3, Section 2, proof of Proposition 2.11]). Let $\bar{f}: C_{\mathrm{c}}^{\infty}\left(\Omega_{1}, \mathbf{E}_{s}^{2}\right) \rightarrow \mathbb{R}$ be given by $\bar{f}\left(\boldsymbol{\varphi}_{2}^{*}\right) \equiv$ $\widehat{f}\left(\boldsymbol{\varphi}_{2 \mid \bar{\Omega}}^{*}, \beta_{0}\left(\boldsymbol{\varphi}_{2 \mid \bar{\Omega}}^{*}\right)\right)$ for $\boldsymbol{\varphi}_{2}^{*} \in C_{\mathrm{c}}^{\infty}\left(\Omega_{1}, \mathbf{E}_{s}^{2}\right)$, where $\Omega \subset \subset \Omega_{1}$. Then, by (4.59), for every $\boldsymbol{\varphi}_{1}^{*} \in C_{\mathrm{c}}^{\infty}\left(\Omega_{1}, \mathbf{E}_{s}^{2}\right)$ such that $\operatorname{div} \operatorname{div} \varphi_{1}^{*}=0$ on $\Omega_{1}$, we get

$$
\begin{aligned}
\bar{f}\left(\boldsymbol{\varphi}_{1}^{*}\right)= & \int_{\Omega} \boldsymbol{\varphi}_{1}^{*}: \widetilde{\varkappa} d x-\int_{\operatorname{Fr} \Omega} \boldsymbol{\varphi}_{1}^{*}:\left(\boldsymbol{\nu} \otimes \boldsymbol{\nu} \gamma_{1}(\widehat{u})\right) d s \\
& +\left\langle\beta_{0}\left(\boldsymbol{\varphi}_{1 \mid \bar{\Omega}}^{*}\right) ; \gamma_{0}(\widehat{u})\right\rangle_{\left(\gamma_{0}\left(W^{2,1}\right)\right)^{*} \times \gamma_{0}\left(W^{2,1}\right)} \\
= & \left\langle\left(\widetilde{\varkappa},\left(\boldsymbol{\nu} \otimes \boldsymbol{\nu} \gamma_{1}(\widehat{u})\right), \gamma_{0}(\widehat{u})\right) ; \boldsymbol{\varphi}_{1}^{*}\right\rangle_{D^{\prime}\left(\Omega_{1}\right) \times D\left(\Omega_{1}\right)}=0 .
\end{aligned}
$$

Therefore, by Proposition 1 , there exists $\widetilde{u}_{1} \in D^{\prime}\left(\Omega_{1}\right)$ such that $\nabla \nabla \widetilde{u}_{1}=$ $\left(\widetilde{\varkappa},\left(\boldsymbol{\nu} \otimes \boldsymbol{\nu} \gamma_{1}(\widehat{u})\right), \gamma_{0}(\widehat{u})\right) \in D^{\prime}\left(\Omega_{1}\right)$.

Step 2. By (4.60) we can assume that $\operatorname{supp} \widetilde{u}_{1} \subset \bar{\Omega}$. For every $\varphi_{3}^{*} \in$ $C_{\mathrm{c}}^{\infty}\left(\Omega, \mathbf{E}_{s}^{2}\right)$ we have $\left\langle\nabla \nabla \widetilde{u}_{1} ; \boldsymbol{\varphi}_{3}^{*}\right\rangle_{D^{\prime}\left(\Omega_{1}\right) \times D\left(\Omega_{1}\right)}=\int_{\Omega} \boldsymbol{\varphi}_{3}^{*}: \tilde{\varkappa} d x$, since $C_{\mathrm{c}}^{\infty}\left(\Omega, \mathbf{E}_{s}^{2}\right)$ $\subset C_{\mathrm{c}}^{\infty}\left(\Omega_{1}, \mathbf{E}_{s}^{2}\right)$. Then $\nabla \nabla \widetilde{u}_{1}(x)=\widetilde{\varkappa}(x)$ for $d x$-a.e. $x \in \Omega$. By [14, Chap. 3, (2.79)], $\widetilde{u}_{1 \mid \Omega} \in W^{2,1}(\Omega)$, because $\tilde{\varkappa} \in L^{\infty}\left(\Omega, \mathbf{E}_{s}^{2}\right)$.

Step 3. Let $\widetilde{u}_{2}$ be the projection of $\widetilde{u}_{1 \mid \Omega}$ onto $W^{2,1}(\Omega) / \operatorname{ker} \nabla \nabla(\cdot)$, given by $\widetilde{u}_{2}=\widetilde{u}_{1 \mid \Omega}-\left(P_{I}\left(\widetilde{u}_{1 \mid \Omega}\right)+P_{I I}\left(\widetilde{u}_{1 \mid \Omega}\right)+P_{I I I}\left(\widetilde{u}_{1 \mid \Omega}\right)\right)$ (cf. (4.51)-(4.53)). By Lemma 20 and Green's formula (2.17) we obtain

$$
\begin{aligned}
(4.61)= & \int_{\Omega} \mathbf{M}: \nabla \nabla \widetilde{u}_{2} d x \\
& -\int_{\operatorname{Fr} \Omega} \beta_{1}(\mathbf{M}) \gamma_{1}(\widehat{u}) d s+\left\langle\beta_{0}(\mathbf{M}) ; \gamma_{0}(\widehat{u})\right\rangle_{\left(\gamma_{0}\left(W^{2,1}\right)\right)^{*} \times \gamma_{0}\left(W^{2,1}\right)} \\
= & -\int_{\operatorname{Fr} \Omega} \beta_{1}(\mathbf{M})\left(\gamma_{1}(\widehat{u})-\gamma_{1}\left(\widetilde{u}_{2}\right)\right) d s \\
& +\left\langle\beta_{0}(\mathbf{M}) ;\left(\gamma_{0}(\widehat{u})-\gamma_{0}\left(\widetilde{u}_{2}\right)\right)\right\rangle_{\left(\gamma_{0}\left(W^{2,1}\right)\right)^{*} \times \gamma_{0}\left(W^{2,1}\right)}
\end{aligned}
$$

for every $\mathbf{M} \in W_{\text {div }}^{2}\left(\Omega\right.$, div div). Then there exists $\widetilde{u}_{3} \in \operatorname{ker} \nabla \nabla(\cdot)$ such that $\gamma_{1}\left(\widetilde{u}_{2}+\widetilde{u}_{3}\right)=\gamma_{1}(\widehat{u})$ and $\gamma_{0}\left(\widetilde{u}_{2}+\widetilde{u}_{3}\right)=\gamma_{0}(\widehat{u})$. Therefore $\widetilde{u}=\widetilde{u}_{2}+\widetilde{u}_{3}$ satisfies (4.58). 
Proposition 22. Let $r>0$ (in the definition of $H_{r}, c f .(4.23)$ ) and let the space $W_{\mathrm{div}}^{2}(\Omega, \operatorname{div} \operatorname{div})$ be defined by (2.12). Then

$$
H_{r}^{*}(\mathbf{0}, 0)=\inf \left\{\widetilde{H}_{r}^{*}\left(\boldsymbol{\varphi}_{s}^{*}, \beta_{0}\left(\boldsymbol{\varphi}_{s}^{*}\right)\right) \mid \boldsymbol{\varphi}_{s}^{*} \in W_{\text {div }}^{2}(\Omega, \operatorname{div} \operatorname{div})\right\} .
$$

Proof. Step 1. Suppose there exists $\delta_{1}>0$ such that

$$
H_{r}^{*}(\mathbf{0}, 0)+\delta_{1}<\inf \left\{\widetilde{H}_{r}^{*}\left(\boldsymbol{\varphi}_{s}^{*}, \beta_{0}\left(\boldsymbol{\varphi}_{s}^{*}\right)\right) \mid \boldsymbol{\varphi}_{s}^{*} \in W_{\operatorname{div}}^{2}(\Omega, \operatorname{div} \operatorname{div})\right\} .
$$

In view of Lemmas 16 and 19, it suffices to show that this assumption leads to a contradiction.

Let $\widetilde{H}_{r \mid L^{\infty}}: L^{\infty}\left(\Omega, \mathbf{E}_{s}^{2}\right) \times L^{\infty}\left(\Gamma_{1}\right) \times \gamma_{0}\left(W^{2,1}(\Omega)\right)_{\mid \Gamma_{1}} \rightarrow \mathbb{R} \cup\{+\infty\}$ be the restriction of $\widetilde{H}_{r}$, given by $\widetilde{H}_{r \mid L^{\infty}}(\mathbf{z}, h, w)=\widetilde{H}_{r}(\mathbf{z}, h, w)$ for $\mathbf{z} \in L^{\infty}\left(\Omega, \mathbf{E}_{s}^{2}\right)$, $h \in L^{\infty}\left(\Gamma_{1}, \mathbb{R}\right)$ and $w \in \gamma_{0}\left(W^{2,1}(\Omega)\right)$ such that $w_{\mid \Gamma_{0}}=0$ (cf. (4.45)). Define the dual functional to $\widetilde{H}_{r \mid L^{\infty}}$ by

$$
\begin{array}{r}
\widetilde{H}_{r \mid L^{\infty}}^{*}\left(\boldsymbol{\varphi}^{*}, \psi^{*}\right)=\sup \left\{\int_{\Omega} \boldsymbol{\varphi}^{*}: \mathbf{z} d x-\int_{\operatorname{Fr} \Omega} \boldsymbol{\beta}_{1}\left(\boldsymbol{\varphi}^{*}\right) h d s\right. \\
+\left\langle\psi^{*} ; w\right\rangle_{\left(\gamma_{0}\left(W^{2,1}\right)\right)^{*} \times \gamma_{0}\left(W^{2,1}\right)}-\widetilde{H}_{r \mid L^{\infty}}(\mathbf{z}, h, w) \\
\left.\mathbf{z} \in L^{\infty}\left(\Omega, \mathbf{E}_{s}^{2}\right), h \in L^{\infty}\left(\Gamma_{1}\right), w \in \gamma_{0}\left(W^{2,1}\right) \text { and } w_{\mid \Gamma_{0}}=0\right\}
\end{array}
$$

for $\left(\varphi^{*}, \psi^{*}\right) \in A(\Omega)$. By (4.34) and (4.38) we obtain $\widetilde{H}_{r \mid L^{\infty}}^{*}\left(\varphi^{*}, \psi^{*}\right)=$ $\widetilde{H}_{r}^{*}\left(\boldsymbol{\varphi}^{*}, \psi^{*}\right)$ for every $\left(\boldsymbol{\varphi}^{*}, \psi^{*}\right) \in A(\Omega)$. Therefore $H_{r}^{*}$ is 1.s.c. on $A(\Omega)$ in the topology (4.56).

Step 2. The linear space $W_{\text {div }}^{2}\left(\Omega\right.$, div div) is closed in $L^{\infty}\left(\Omega, \mathbf{E}_{s}^{2}\right)$ endowed with the topology $\sigma\left(L^{\infty}\left(\Omega, \mathbf{E}_{s}^{2}\right) ; L^{\infty}\left(\Omega, \mathbf{E}_{s}^{2}\right)\right)$. Indeed, let $\left\{\mathbf{M}_{k}\right\}_{k \in K}$ $\subset W_{\mathrm{div}}^{2}(\Omega, \operatorname{div} \operatorname{div})$ be a net convergent to $\mathbf{M}_{0}$ in $\sigma\left(L^{\infty}\left(\Omega, \mathbf{E}_{s}^{2}\right) ; L^{\infty}\left(\Omega, \mathbf{E}_{s}^{2}\right)\right)$, i.e. $\int_{\Omega}\left(\mathbf{M}_{k}-\mathbf{M}_{0}\right): \mathbf{p} d x \rightarrow 0$ for every $\mathbf{p} \in L^{\infty}\left(\Omega, \mathbf{E}_{s}^{2}\right)$. Then for every $u \in C_{\mathrm{c}}^{2}(\Omega, \mathbb{R})$,

(4.65) $0=\int_{\Omega}\left(\operatorname{div} \operatorname{div} \mathbf{M}_{k}\right) u d x=\int_{\Omega} \mathbf{M}_{k}:(\nabla \nabla u) d x \rightarrow \int_{\Omega} \mathbf{M}_{0}:(\nabla \nabla u) d x$.

Therefore $\operatorname{div} \operatorname{div} \mathbf{M}_{0}=0$ in the sense of distributions on $\Omega$.

Step 3. The set $W_{\text {div }}^{2}(\Omega, \operatorname{div} \operatorname{div}) \cap B_{L^{\infty}\left(\Omega, \mathbf{E}_{s}^{2}\right)}(0, \widehat{r})$ endowed with the topology (4.56) is compact.

Indeed, let $\left\{\mathbf{M}_{k}\right\}_{k \in K} \subset W_{\operatorname{div}}^{2}(\Omega, \operatorname{div} \operatorname{div}) \cap B_{L^{\infty}\left(\Omega, \mathbf{E}_{s}^{2}\right)}(0, \widehat{r})$ be a net. Then there exists a finer net $\left\{\mathbf{M}_{k_{t}}\right\}_{t \in T}$ and $\mathbf{M}_{0} \in W_{\operatorname{div}}^{2}(\Omega, \operatorname{div} \operatorname{div}) \cap B_{L^{\infty}\left(\Omega, \mathbf{E}_{s}^{2}\right)}(0, \widehat{r})$ such that $\left\{\mathbf{M}_{k_{t}}\right\}_{t \in T}$ converges to $\mathbf{M}_{0}$ in $\sigma\left(L^{\infty}\left(\Omega, \mathbf{E}_{s}^{2}\right) ; L^{\infty}\left(\Omega, \mathbf{E}_{s}^{2}\right)\right)$, because the Hausdorff topology $\sigma\left(L^{\infty} ; L^{\infty}\right)$ is weaker than $\sigma\left(L^{\infty} ; L^{1}\right)$ (cf. [11, Corollary 3.1.14]). The trace $\left(\beta_{0}, \beta_{1}\right)$ is a continuous linear map from $\left[W^{2}(\Omega, \operatorname{div} \operatorname{div}),\|\cdot\|_{W^{2}(\Omega, \operatorname{div} \operatorname{div})}\right]$ into $\left[\left(\gamma_{0}\left(W^{2,1}(\Omega)\right)\right)^{*},\|\cdot\|_{0^{*}}\right] \times\left[L^{\infty}(\operatorname{Fr} \Omega)\right.$, $\left.\|\cdot\|_{L^{\infty}}\right]$. Hence the net $\left\{\left(\beta_{0}\left(\mathbf{M}_{k_{t}}\right), \beta_{1}\left(\mathbf{M}_{k_{t}}\right)\right)\right\}_{t \in T}$ is bounded in $\left(\gamma_{0}\left(W^{2,1}(\Omega)\right)\right)^{*}$ 
$\times L^{\infty}(\operatorname{Fr} \Omega)$. Therefore there exists a finer net $\left\{\mathbf{M}_{k_{t_{p}}}\right\}_{p \in P}$ and $\left(\widetilde{\varrho}_{0}, \widetilde{\varrho}_{1}\right) \in$ $\left(\gamma_{0}\left(W^{2,1}(\Omega)\right)\right)^{*} \times L^{\infty}(\operatorname{Fr} \Omega)$ such that

$$
\int_{\operatorname{Fr} \Omega}\left(\beta_{1}\left(\mathbf{M}_{k_{t_{p}}}\right)-\widetilde{\varrho}_{1}\right) h d s-\left\langle\left(\beta_{0}\left(\mathbf{M}_{k_{t_{p}}}\right)-\widetilde{\varrho}_{0}\right) ; w\right\rangle_{\left(\gamma_{0}\left(W^{2,1}\right)\right)^{*} \times \gamma_{0}\left(W^{2,1}\right)} \rightarrow 0
$$

for every $(h, w) \in L^{1}(\operatorname{Fr} \Omega) \times \gamma_{0}\left(W^{2,1}(\Omega)\right)$. Moreover, by (2.17), similarly to Step 4 of the proof of [4, Proposition 19] we obtain $\beta_{0}\left(\mathbf{M}_{0}\right)=\widetilde{\varrho}_{0}$ and $\beta_{1}\left(\mathbf{M}_{0}\right)=\widetilde{\varrho}_{1}$.

Step 4. By the Hahn-Banach theorem, for every $m \in \mathbb{N}$, there exists an affine functional $A(\Omega) \ni\left(\varphi^{*}, \psi^{*}\right) \mapsto \widetilde{f}_{m}\left(\varphi^{*}, \psi^{*}\right)+\widetilde{a}_{m} \in \mathbb{R}$ such that

$$
\widetilde{f}_{m}\left(\varphi^{*}, \psi^{*}\right)+\widetilde{a}_{m}<\widetilde{H}_{r}^{*}\left(\varphi^{*}, \psi^{*}\right)
$$

for $\left(\varphi^{*}, \psi^{*}\right) \in A(\Omega)$,

$$
\begin{aligned}
\widetilde{f}_{m}\left(\widetilde{\mathbf{M}}_{s}, \beta_{0}\left(\widetilde{\mathbf{M}}_{s}\right)\right)+\widetilde{a}_{m}>\inf _{\varphi_{s}^{*}}\left\{\widetilde{H}_{r}^{*}\left(\boldsymbol{\varphi}_{s}^{*}, \beta_{0}\left(\boldsymbol{\varphi}_{s}^{*}\right)\right) \mid\right. \\
\left.\boldsymbol{\varphi}_{s}^{*} \in W_{\mathrm{div}}^{2}(\Omega, \operatorname{div} \operatorname{div})\right\}-\delta_{1} / 2^{m}
\end{aligned}
$$

for $\widetilde{\mathbf{M}}_{s} \in W_{\text {div }}^{2}(\Omega, \operatorname{div} \operatorname{div}) \cap B_{L^{\infty}\left(\Omega, \mathbf{E}_{s}^{2}\right)}\left(0, \widehat{r} 2^{m}\right)$ and $\widetilde{f}_{m}$ is continuous in the topology (4.56). Indeed, $\widetilde{H}_{r}^{*}$ is l.s.c. and $W_{\mathrm{div}}^{2}(\Omega, \operatorname{div} \operatorname{div}) \cap B_{L^{\infty}\left(\Omega, \mathbf{E}_{s}^{2}\right)}\left(0, \widehat{r} 2^{m}\right)$ is compact in this topology. By (4.37) and Assumption $4, \widetilde{H}_{r}^{*}(\mathbf{0}, 0)<\infty$. Because of (4.21), (4.22) and Assumption 4,

$$
\widetilde{H}_{r}^{*}(\mathbf{0}, 0)=\inf \left\{\widetilde{H}_{r}^{*}\left(\boldsymbol{\varphi}_{s}^{*}, \beta_{0}\left(\boldsymbol{\varphi}_{s}^{*}\right)\right) \mid \boldsymbol{\varphi}_{s}^{*} \in W_{\operatorname{div}}^{2}(\Omega, \operatorname{div} \operatorname{div})\right\} .
$$

Step 5. Due to Steps 5, 6 and 7 of the proof of [4, Proposition 19] we obtain the existence of $\widetilde{u} \in W^{2,1}(\Omega)$ such that $\gamma_{1}(\widetilde{u})=0=\gamma_{0}(\widetilde{u})$ on $\Gamma_{0}$, $\nabla \nabla \widetilde{u} \in L^{\infty}\left(\Omega, \mathbf{E}_{s}^{2}\right), \gamma_{1}(\widetilde{u}) \in L^{\infty}(\operatorname{Fr} \Omega)$ and

$$
\begin{aligned}
A(\Omega) \ni\left(\boldsymbol{\varphi}^{*}, \psi^{*}\right) & \mapsto \widetilde{f}_{0}\left(\boldsymbol{\varphi}^{*}, \psi^{*}\right)=\int_{\Omega} \boldsymbol{\varphi}^{*}:(\nabla \nabla \widetilde{u}) d x \\
& -\int_{\operatorname{Fr} \Omega} \beta_{1}\left(\boldsymbol{\varphi}^{*}\right) \gamma_{1}(\widetilde{u}) d s+\left\langle\psi^{*} ; \gamma_{0}(\widetilde{u})\right\rangle_{\left(\gamma_{0}\left(W^{2,1}\right)\right)^{*} \times \gamma_{0}\left(W^{2,1}\right)}
\end{aligned}
$$

satisfies the inequalities

$$
\widetilde{f}_{0}\left(\varphi^{*}, \psi^{*}\right)+\widetilde{H}_{r}^{*}(\mathbf{0}, 0)-\delta_{1} / 2<\widetilde{H}_{r}^{*}\left(\boldsymbol{\varphi}^{*}, \psi^{*}\right), \quad \widetilde{f}_{0}\left(\widetilde{\mathbf{M}}_{s}, \beta_{0}\left(\widetilde{\mathbf{M}}_{s}\right)\right) \geq 0
$$
for every $\left(\varphi^{*}, \psi^{*}\right) \in A(\Omega)$ and every $\widetilde{\mathbf{M}}_{s} \in W_{\text {div }}^{2}(\Omega$, div div) (cf. Lemma 21 ).

Step 6. We say that a net $\left\{\left(\varphi_{k}^{*}, \psi_{k}^{*}\right)\right\}_{k \in K} \subset C_{\text {div div }}^{1}\left(\bar{\Omega}, \mathbf{E}_{s}^{2}\right) \times C(\operatorname{Fr} \Omega)$ converges to $\left(\widehat{\boldsymbol{\varphi}}^{*}, \widehat{\psi}^{*}\right)$ in the topology $\sigma\left(C_{\operatorname{div} \operatorname{div}}^{1}\left(\bar{\Omega}, \mathbf{E}_{s}^{2}\right) \times C(\operatorname{Fr} \Omega) ; W^{2,1}(\Omega)\right)$ if

$$
\left\langle\left(\nabla \nabla u, \gamma_{1}(u), \gamma_{0}(u)\right) ;\left(\left(\boldsymbol{\varphi}_{k}^{*}-\widehat{\varphi}^{*}\right),\left(\psi_{k}^{*}-\widehat{\psi}^{*}\right)\right)\right\rangle_{1} \rightarrow 0
$$

for every $u \in W^{2,1}(\Omega)$ such that $\gamma_{1}(u)_{\mid \Gamma_{0}}=0$ on $\Gamma_{0}$ (see (4.33)). The l.s.c. regularization of $\widetilde{H}_{r}^{*}$ in the topology $\sigma\left(C_{\operatorname{div} \operatorname{div}}^{1}\left(\bar{\Omega}, \mathbf{E}_{s}^{2}\right) \times C(\operatorname{Fr} \Omega) ; W^{2,1}(\Omega)\right)$ 
(denoted by $\mathrm{cl}_{(4.71)} \widetilde{H}_{r}^{*}$ ) is given by

$$
\begin{aligned}
& \operatorname{cl}_{(4.71)} \widetilde{H}_{r}^{*}\left(\varphi^{*}, \psi^{*}\right)=\sup \left\{\left\langle\left(\nabla \nabla u, \gamma_{1}(u), \gamma_{0}(u)\right) ;\left(\varphi^{*}, \psi^{*}\right)\right\rangle_{1}\right. \\
&\left.\quad-\widetilde{H}_{r}^{* \#}\left(\nabla \nabla u, \gamma_{1}(u), \gamma_{0}(u)\right) \mid u \in W^{2,1}(\Omega), \gamma_{1}(u)_{\mid \Gamma_{0}}=0\right\} \\
&= \sup \left\{\left\langle\left(\nabla \nabla u, \gamma_{1}(u), \gamma_{0}(u)\right) ;\left(\varphi^{*}, \psi^{*}\right)\right\rangle_{1}\right. \\
&\left.\quad-H_{r}\left(\nabla \nabla u, \gamma_{1}(u), \gamma_{0}(u)\right) \mid u \in W^{2,1}(\Omega), \gamma_{1}(u)_{\mid \Gamma_{0}}=0\right\} \\
&= H_{r}^{*}\left(\varphi^{*}, \psi^{*}\right),
\end{aligned}
$$

for every $\left(\boldsymbol{\varphi}^{*}, \psi^{*}\right) \in C_{\operatorname{div} \operatorname{div}}^{1}\left(\bar{\Omega}, \mathbf{E}_{s}^{2}\right) \times C(\operatorname{Fr} \Omega)$ (cf. Proposition 17 and Lemma 18). From (4.63), (4.69), (4.70) and (4.72) we obtain a contradiction.

Proposition 23. For every $r>0$,

$$
\inf \left\{H_{r}(\overline{\mathbf{z}}) \mid \overline{\mathbf{z}} \in \mathcal{Z}(H B(\Omega))\right\}=\inf \left\{\widetilde{H}_{r}(\overline{\mathbf{z}}) \mid \overline{\mathbf{z}} \in \widetilde{\mathcal{Z}}(H B(\Omega))\right\} .
$$

Proof. By (4.35), (4.37), (4.21), (4.22), Assumption 4, (4.37), Proposition 22 , and (4.30), we get

$$
\begin{aligned}
\sup \{ & \left.-\widetilde{H}_{r}(\overline{\mathbf{z}}) \mid \overline{\mathbf{z}} \in \mathcal{Z}(H B(\Omega))\right\}=\widetilde{H}_{r}^{*}(\mathbf{0}, 0) \\
& =-\left\lceil P_{\lambda, r}^{*}\right\rceil\left(\mathbf{M}_{L}\right)=\inf \left\{-\left\lceil P_{\lambda, r}^{*}\right\rceil(\mathbf{M}) \mid \mathbf{M} \in W^{2}(\Omega, \operatorname{div} \operatorname{div})\right\} \\
& =\inf \left\{\widetilde{H}_{r}^{*}\left(\mathbf{M}_{s}, \beta_{0}\left(\mathbf{M}_{s}\right)\right) \mid \mathbf{M}_{s} \in W_{\operatorname{div}}^{2}(\Omega, \operatorname{div} \operatorname{div})\right\} \\
& =H_{r}^{*}(\mathbf{0}, 0)=\sup \left\{-H_{r}\left(\nabla \nabla u, \gamma_{1}(u), \gamma_{0}(u)\right) \mid u \in H B(\Omega)\right\}
\end{aligned}
$$

Let

$$
\mathrm{cl}_{\sigma\left(\mathcal{Z}(H B) ;\left[C \cap W^{2}\right] \times C\right)} H_{r} \quad\left(\text { resp. } \operatorname{cl}_{\sigma\left(\widetilde{\mathcal{Z}}(H B) ;\left[C \cap W^{2}\right] \times C\right)} \widetilde{H}_{r}\right)
$$

denote the largest l.s.c. minorant of $H_{r}$ in the topology $\sigma(\mathcal{Z}(H B(\Omega))$; $C_{\text {div div }}^{1}\left(\bar{\Omega}, \mathbf{E}_{s}^{2}\right) \times C(\operatorname{Fr} \Omega)$ ) (respectively, the largest l.s.c. minorant of $\widetilde{H}_{r}$ in $\left.\sigma\left(\widetilde{\mathcal{Z}}(H B(\Omega)) ; C_{\operatorname{div} \operatorname{div}}^{1}\left(\bar{\Omega}, \mathbf{E}_{s}^{2}\right) \times C(\operatorname{Fr} \Omega)\right)\right)$, i.e. (4.75) stands for the l.s.c. regularizations of $H_{r}$ and $\widetilde{H}_{r}$ in the above mentioned topologies.

Because $(\mathbf{0}, 0) \in C_{\operatorname{div} \operatorname{div}}^{1}\left(\bar{\Omega}, \mathbf{E}_{s}^{2}\right) \times C(\operatorname{Fr} \Omega)$ and from Proposition 23 we get

$$
\begin{aligned}
& \inf \left\{\operatorname{cl}_{\sigma\left(\mathcal{Z}(H B) ;\left[C \cap W^{2}\right] \times C\right)} H_{r}(\overline{\mathbf{z}}) \mid \overline{\mathbf{z}} \in \mathcal{Z}(H B(\Omega))\right\} \\
& \quad=\inf \left\{\operatorname{cl}_{\sigma\left(\widetilde{\mathcal{Z}}(H B) ;\left[C \cap W^{2}\right] \times C\right)} \widetilde{H}_{r}(\overline{\mathbf{z}}) \mid \overline{\mathbf{z}} \in \widetilde{\mathcal{Z}}(H B(\Omega))\right\} .
\end{aligned}
$$

The main result of this paper is the following.

THEOREM 24. Let $r>0$ and suppose Assumptions 2, 4 and 5 hold. If $H_{r}$ is a coercive function, then by Proposition 17 the functional $H_{r}^{* *}$ is given by (4.39), since $H_{r}^{* *}=\widetilde{H}_{r}^{* \#}$. Moreover, every minimum point $\widehat{\mathbf{z}} \in \mathcal{Z}(H B(\Omega))$ of $H_{r}^{* *}$ is given by a function $\widehat{u} \in W^{2,1}(\Omega)$ such that $\gamma_{1}(\widehat{u})=0$ on $\Gamma_{0}$ and $\widehat{\mathbf{z}}=\left(\nabla \nabla \widehat{u}, \gamma_{1}(\widehat{u}), \gamma_{0}(\widehat{u})\right)$. 
Proof. Step 1. Let $\widehat{\mathbf{z}}=\left(\nabla \nabla \widehat{u}, \gamma_{1}(\widehat{u}), \gamma_{0}(\widehat{u})\right) \in \mathcal{Z}(H B(\Omega))$ be a minimum point of $H_{r}^{* *}$. By (4.30), (4.31), Theorem 9 and Proposition 15 the functional $H_{r}^{* *}$ is the l.s.c. regularization of $H_{r}$ in $\sigma\left(\widetilde{\mathcal{Z}}(H B(\Omega)) ; C_{\operatorname{div} \operatorname{div}}^{1}\left(\bar{\Omega}, \mathbf{E}_{s}^{2}\right) \times\right.$ $C(\operatorname{Fr} \Omega))$. Then, by (4.76), we obtain

$$
\begin{aligned}
H_{r}^{* *}(\widehat{\mathbf{z}}) & =\operatorname{cl}_{\sigma\left(\mathcal{Z}(H B) ;\left[C \cap W^{2}\right] \times C\right)} H_{r}(\widehat{\mathbf{z}}) \\
& =\inf \left\{\operatorname{cl}_{\sigma\left(\widetilde{\mathcal{Z}}(H B) ;\left[C \cap W^{2}\right] \times C\right)} \widetilde{H}_{r}(\overline{\mathbf{z}}) \mid \overline{\mathbf{z}} \in \widetilde{\mathcal{Z}}(H B(\Omega))\right\} .
\end{aligned}
$$

For every $\overline{\mathbf{z}} \in \mathcal{Z}(H B(\Omega))$ we have $H_{r}(\overline{\mathbf{z}})=\widetilde{H}_{r}(\overline{\mathbf{z}})$, hence

$$
\operatorname{cl}_{\sigma\left(\mathcal{Z}(H B) ;\left[C \cap W^{2}\right] \times C\right)} H_{r}(\overline{\mathbf{z}}) \geq \operatorname{cl}_{\sigma\left(\mathcal{Z}(H B) ;\left[C \cap W^{2}\right] \times C\right)} \widetilde{H}_{r}(\overline{\mathbf{z}}) .
$$

Because of (4.77) and (4.76), $\widehat{\mathbf{z}} \in \mathcal{Z}(H B(\Omega))$ is a minimum point of $\operatorname{cl}_{\sigma\left(\mathcal{Z}(H B) ;\left[C \cap W^{2}\right] \times C\right)} \widetilde{H}_{r}$ on $\widetilde{\mathcal{Z}}(H B(\Omega))$. By [10, Chap. 1, (5.2)], $(\mathbf{0}, 0) \in$ $\partial\left(\operatorname{cl}_{\sigma\left(\widetilde{\mathcal{Z}}(H B) ;\left[C \cap W^{2}\right] \times C\right)} \widetilde{H}_{r}\right)(\widehat{\mathbf{z}})$, where $\partial$ is a subgradient and $(\mathbf{0}, 0) \in$ $C_{\text {div div }}^{1}\left(\bar{\Omega}, \mathbf{E}_{s}^{2}\right) \times C(\operatorname{Fr} \Omega)$. Then $\widehat{\mathbf{z}} \in \partial\left(\operatorname{cl}_{\sigma\left(\widetilde{\mathcal{Z}}(H B) ;\left[C \cap W^{2}\right] \times C\right)} \widetilde{H}_{r}\right)^{*}(\mathbf{0}, 0)$ (see [10, Chap. 1, Corollary 5.2]). By [10, Chap. 1, Corollary 4.1] we have $\widehat{\mathbf{z}} \in$ $\partial\left(\widetilde{H}_{r}^{*}\right)(\mathbf{0}, 0)$. Then by [10, Chap. 1, (5.2)] we get

$$
\left\langle\left(\nabla \nabla \widehat{u}, \gamma_{1}(\widehat{u}), \gamma_{0}(\widehat{u})\right) ;\left(\left(\boldsymbol{\varphi}^{*}, \psi^{*}\right)-(\mathbf{0}, 0)\right)\right\rangle_{1}+\widetilde{H}_{r}^{*}(\mathbf{0}, 0) \leq \widetilde{H}_{r}^{*}\left(\boldsymbol{\varphi}^{*}, \psi^{*}\right)
$$
for every $\left(\boldsymbol{\varphi}^{*}, \psi^{*}\right) \in C_{\operatorname{div} \operatorname{div}}^{1}\left(\bar{\Omega}, \mathbf{E}_{s}^{2}\right) \times C(\operatorname{Fr} \Omega)$, since $\widehat{\mathbf{z}}=\left(\nabla \nabla \widehat{u}, \gamma_{1}(\widehat{u}), \gamma_{0}(\widehat{u})\right)$.

Step 2. Because of Assumption $3, \Gamma_{1}=\operatorname{Fr} \Omega \cap \mathcal{C}$, where $\mathcal{C}=\operatorname{clint} \mathcal{C} \subset$ $\Omega_{1}$ is a closed Caccioppoli set, $d s(\operatorname{Fr} \Omega \cap \operatorname{Fr} \mathcal{C})=0$ and $\Omega \subset \subset \Omega_{1}$. Let $\mathcal{O}_{\Gamma_{0}} \equiv \Omega_{1}-\mathcal{C}$. Then $\operatorname{Fr} \Omega \cap \mathcal{O}_{\Gamma_{0}}=\Gamma_{0}$. Therefore for every $k \in \mathbb{N}$ there exists an open set $\Omega_{k}^{\prime}$ such that $\Omega_{k}^{\prime} \subset \mathcal{O}_{\Gamma_{0}}, \Omega_{k}^{\prime} \subset \subset \Omega_{1}, d x\left(\Omega_{k}^{\prime}\right)<1 /(2 k)$ and $\left\{x \in \Gamma_{0} \mid \gamma_{1}(\widehat{u})(x) \neq 0\right\} \subset \Omega_{k}^{\prime}$.

Step 3. Suppose the singular part $(\nabla \nabla \widehat{u})_{s}$ is not 0 or $d s\left(\left\{x \in \Gamma_{0} \mid\right.\right.$ $\left.\left.\gamma_{1}(\widehat{u})(x) \neq 0\right\}\right)>0$. Then there exists $\zeta>0$ such that $\left\|(\nabla \nabla \widehat{u})_{s}\right\|_{\mathbb{M}_{b}}+$ $\int_{\Gamma_{0}}\left|\gamma_{1}(\widehat{u})(x)\right| d s>\zeta$. Therefore, for every $k \in \mathbb{N}$, there exist open sets $\Omega_{k}^{\prime \prime} \subset \subset \Omega$ with $\Omega_{k}^{0} \equiv \Omega_{k}^{\prime \prime} \cup \Omega_{k}^{\prime} \subset \subset \Omega_{1}$ such that $d x\left(\Omega_{k}^{0}\right)<1 / k$ and $\left\|(\nabla \nabla \widehat{u})_{s}\right\|_{\mathbb{M}_{b}\left(\Omega_{k}^{\prime \prime}, \mathbf{E}_{s}^{2}\right)}+\int_{\Gamma_{0}}\left|\gamma_{1}(\widehat{u})(x)\right| d s>\frac{1}{2} \zeta$. The existence of the sequence $\left\{\Omega_{k}^{\prime \prime}\right\}_{k \in \mathbb{N}}$ follows from the regularity of the measure $\nabla \nabla \widehat{u}$. By Assumption 4, $B_{\mathbf{E}_{s}^{2}}\left(\mathbf{M}_{L}(x), \delta_{0}\right) \subset \mathcal{K}_{p}(x)$ for every $x \in \Omega$. Then for every $k \in \mathbb{N}$ there exists $\boldsymbol{\varphi}_{k}^{*} \in C_{\mathrm{c}}^{2}\left(\Omega_{1}, \mathbf{E}_{s}^{2}\right)$ such that $\boldsymbol{\varphi}_{k \mid \Omega_{1}-\Omega_{k}^{0}}^{*}=0$,

$$
\begin{gathered}
\left\|\boldsymbol{\varphi}_{k}^{*}(x)\right\|_{\mathbf{E}_{s}^{2}}<\frac{1}{2} \delta_{0} \quad \forall x \in \Omega_{k}^{0}, \\
\left\langle\left(\nabla \nabla \widehat{u}, \gamma_{1}(\widehat{u}), \gamma_{0}(\widehat{u})\right) ;\left(\boldsymbol{\varphi}_{k}^{*}, 0\right)\right\rangle_{1}>\frac{1}{8} \zeta \delta_{0}
\end{gathered}
$$

(cf. Step 3 of the proof of [4, Theorem 21]). Due to Step 4 of the proof of [4, Theorem 21] we obtain the conclusion of Theorem 24 . 


\section{References}

[1] R. S. Adams, Sobolev Spaces, Academic Press, New York, 1975.

[2] J. L. Bojarski, The relaxation of Signorini problems in Hencky plasticity, I: Threedimensional solid, Nonlinear Anal. 29 (1997), 1091-1116.

[3] - , The relaxation of Signorini problems in Hencky plasticity, II: Plates, ibid. 29 (1997), 1117-1143.

[4] -, Regularity of solutions in plasticity. I: Continuum, Appl. Math. (Warsaw) 30 (2003), 337-364.

[5] G. Bouchitté and M. Valadier, Integral representation of convex functionals on a space of measures, J. Funct. Anal. 80 (1988), 398-420.

[6] M. Carriero, A. Leaci and F. Tomarelli, Free gradient discontinuities, in: Calculus of Variations, Homogenization and Continuum Mechanics, G. Bouchitté et al. (eds.), Ser. Adv. Math. Appl. Sc. 18, World Sci., Singapore, 1994.

[7] F. Demengel, Compactness theorems for spaces of functions with bounded derivatives and applications to limit analysis problems in plasticity, Arch. Rat. Mech. Anal. 105 (1989), 123-161.

[8] —, Fonctions à hessien borné, Ann. Inst. Fourier (Grenoble) 34 (1984), no. 2, 155190.

[9] N. Dunford and J. T. Schwartz, Linear Operators, Part I, Interscience, New York, 1958.

[10] I. Ekeland and R. Temam, Convex Analysis and Variational Problems, NorthHolland, Amsterdam and New York, 1976.

[11] R. Engelking, General Topology, PWN-Polish Sci. Publ., Warszawa, 1977.

[12] E. Giusti, Minimal Surfaces and Functions of Bounded Variation, with notes by G. H. Williams, Notes on Pure Math. 10, Dept. Math., Australian National Univ., Canberra, 1977.

[13] R. T. Rockafellar, Integral functionals, normal integrands and measurable selections, in: Nonlinear Operators and the Calculus of Variations, Lecture Notes in Math. 543, Springer, Berlin, 1975, 157-207.

[14] R. Temam, Mathematical Problems in Plasticity, Gauthier-Villars, Paris, 1985.

[15] F. Tomarelli, Special Bounded Hessian and partial regularity of equilibrium for a plastic plate, in: Developments in PDE and Applications to Mathematical Physics, G. Buttazzo et al. (eds.), Plenum Press, New York, 1992, 235-240.

Institute of Fundamental Technological Research

Polish Academy of Sciences

Świętokrzyska 21

00-049 Warszawa, Poland

E-mail: jbojar@ippt.gov.pl

Received on 19.8.2002;

revised version on 1.10.2003 